ARTICLE

DOI: $10.1038 / s 41467-017-02355-w$

\title{
ANGPTL8 negatively regulates NF-kB activation by facilitating selective autophagic degradation of $\mathrm{IKK} \gamma$
}

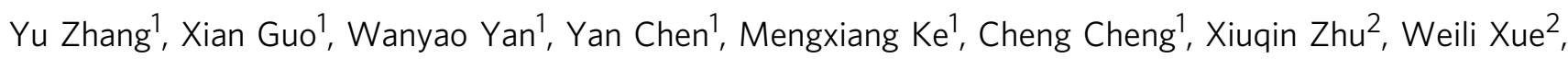
Qiaoqiao Zhou', Ling Zheng ${ }^{2}$, Shun Wang ${ }^{3}$, Bin Wu ${ }^{3}$, Xinran Liu', Liang Ma', Lianqi Huang ${ }^{1}$ \& Kun Huang ${ }^{1,4}$

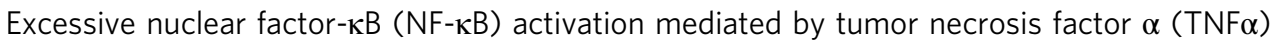
plays a critical role in inflammation. Here we demonstrate that angiopoietin-like 8 (ANGPTL8) functions as a negative feedback regulator in TNF $\alpha$-triggered NF-KB activation intracellularly. Inflammatory stimuli induce ANGPTL8 expression, and knockdown or knockout of ANGPTL8 potentiates TNF $\alpha$-induced NF-KB activation in vitro. Mechanistically, upon TNF $\alpha$ stimulation, ANGPTL8 facilitates the interaction of IKK $\gamma$ with p62 via forming a complex, thus promoting the selective autophagic degradation of IKK $\gamma$. Furthermore, the $\mathrm{N}$ terminal domain mediated self-oligomerization of ANGPTL8 is essential for IKK $\gamma$ degradation and NF-KB activation. In vivo, circulating ANGPTL8 level is high in patients diagnosed with infectious diseases, and the ANGPTL8/p62-IKK $\gamma$ axis is responsive to inflammatory stimuli in the liver of LPS-injected mice. Altogether, our study suggests the ANGPTL8/p62-IKK $\gamma$ axis as a negative feedback loop that regulates NF-KB activation, and extends the role of selective autophagy in fine-tuned inflammatory responses.

\footnotetext{
${ }^{1}$ Tongji School of Pharmacy, Huazhong University of Science \& Technology, Wuhan 430030, China. ${ }^{2}$ Hubei Key Laboratory of Cell Homeostasis, College of Life Sciences, Wuhan University, Wuhan 430072, China. ${ }^{3}$ Department of Blood Transfusion, Wuhan Hospital of Traditional and Western Medicine, Wuhan 430022, China. ${ }^{4}$ Centre for Biomedicine Research, Wuhan Institute of Biotechnology, Wuhan 430075, China. Correspondence and requests for materials should be addressed to K.H. (email: kunhuang2008@hotmail.com)
} 
$\mathrm{N}$ F- $\kappa \mathrm{B}$ plays a pivotal role in a variety of physiological and pathological processes, including inflammation, immunity and metabolism ${ }^{1}$. In non-stimulated cells, NF- $\kappa B$ is kept inactive in cytosol by binding to inhibitor of $\kappa \mathrm{B} \alpha$ (I $\mathrm{I} \mathrm{B} \alpha)$. Many agents, including pro-inflammatory cytokines, cause phosphorylation and degradation of $\mathrm{I} \kappa \mathrm{B} \alpha$, which results in releasing of NF- $\mathrm{BB}$ for translocation to the nucleus and initiating the expression of downstream genes ${ }^{2}$. Among these agentsmediated signaling, TNF $\alpha$ induction is a classical model to study the regulatory mechanisms of NF- $\mathrm{KB}$ activation. TNF $\alpha$ binds to its receptor TNF-RI to recruit the TNFR-associated death domain (TRADD), which recruits TNFR-associated factor 2 (TRAF2), TRAF5, and receptor interacting protein 1 (RIP1) to the receptor complex, then RIP1 further recruits and activates the transforming growth factor $\beta$ activated kinase-1 (TAK1) complex and the IKB kinase (IKK) complex. The IKK complex consists of the catalytic subunits IKK $\alpha$ and IKK $\beta$, and a regulatory subunit IKK $\gamma$. $\mathrm{IKK} \gamma$, also known as NF- $\kappa \mathrm{B}$ essential modulator (NEMO), is critical for the activation of the IKK complex; moreover, IKK $\gamma$ also works as a scaffold which specifically channels the kinase activity of IKK $\beta$ to I $\kappa B \alpha^{3-5}$. Finally, I $\mathrm{B} \alpha$ is phosphorylated and degraded, thereby releasing NF- $\kappa \mathrm{B}$ to the nucleus.

Inappropriate NF- $\kappa \mathrm{B}$ activation may cause immunodeficiency, chronic inflammation, autoimmunity and malignancy ${ }^{6-8}$. Therefore, IKK activation and I $\mathrm{I} B \alpha$ phosphorylation, two key steps in NF- $\kappa \mathrm{B}$ activation, should be tightly regulated, which highlights the physiological significance of IKK $\gamma$. Hypomorphic IKK $\gamma$ mutations (IKKG gene located on the $\mathrm{X}$ chromosome) are lethal for male and lead to immune and developmental defects in female ${ }^{9,10}$; while inactivation of the negative regulators of IKK $\gamma$, such as deubiquitinase A20 and CYLD lysine 63 deubiquitinase (CYLD), leads to serious disorders ${ }^{11,12}$; similarly, males with a "gain of function" IKK $\gamma$ mutant ( $\triangle \mathrm{CT}-\mathrm{NEMO}$, a C-terminal domain truncated mutant), which fails to recruit A20, develop autoinflammatory diseases ${ }^{13}$.

Proteolysis of signaling molecules is an important way to shut down signaling transduction. The ubiquitin-proteasome system (UPS) and autophagy are two major protein degradation pathways $^{14}$. Previously, it was thought that UPS is highly selective while autophagy is a non-selective bulk process ${ }^{14}$. Recent studies suggest that autophagy can also target specific protein aggregates or other substrates for degradation, referred to as selective autophagy ${ }^{15,16}$. In general, proteins enter the selective autophagy are first K63-polyubiquitinated, then bound by one or more of autophagy receptors such as p62, neighbor of BRCA1 gene 1 (NBR1), nuclear dot protein $52 \mathrm{kDa}$ (NDP52), TOLL interacting protein (Tollip), and optineurin (OPTN), followed by engulfment in autophagosomes ${ }^{14,16}$. Increasing evidences suggest that autophagy is important for the inflammation and immune responses $^{17,18}$, however the role of selective autophagy in these critical physiological processes is poorly understood.

ANGPTL8 (also called Lipasin, RIFL, TD26 or C19orf80) is known as a key regulator of plasma lipid metabolism, which functions mainly by inhibiting lipoprotein lipase ${ }^{19,20}$. Here, we demonstrate intracellular ANGPTL8 as a novel negative feedback regulator of TNF $\alpha$-mediated NF- $\mathrm{NB}$ activation, which may work as a critical step to avoid excessive inflammatory responses by facilitating p62-mediated autophagic IKK $\gamma$ degradation.

\section{Results}

Pro-inflammatory cytokines up-regulate ANGPTL8. ANGPTL8 regulates lipid metabolism, and the level of circulating ANGPTL8 is increased in type 2 diabetes (T2D) $)^{21,22}$. Since lipid toxicity and $\mathrm{T} 2 \mathrm{D}$ are tightly correlated with inflammation, we investigated the level of ANGPTL8 upon stimulation of pro-inflammatory cytokines such as TNF $\alpha$ and IL-1 $\beta$. In HepG2 cells, the transcription and expression of ANGPTL8 were both significantly increased after being treated with TNF $\alpha$ (Fig. 1a, b), with a TNF $\alpha$ dose-dependent elevation in ANGPTL8 level (Fig. 1c). Similar results were observed in two additional cell lines, HEK293T (a human embryotic kidney cell line) and A549 (a human lung cancer cell line) (Supplementary Fig. 1a), although their ANGPTL8 level was markedly lower than that of HepG2 cells (Supplementary Fig. 1b). Consistently, IL-1 $\beta$ treatment induced the transcription and expression of ANGPTL8 in HepG2 cells (Supplementary Fig. 1c, d). Collectively, these results indicate that the ANGPTL8 expression could be triggered by different inflammatory stimuli and in various cells.

Knockdown or knockout of ANGPTL8 potentiates NF-кB activation. We next determined whether ANGPTL8 regulates NF- $\kappa \mathrm{B}$ activation. Three ANGPTL8-RNAi plasmids were generated, which efficiently inhibited the transcription and expression of ANGPTL8 in HepG2 cells (Fig. 2a). In luciferase reporter assays, knockdown of ANGPTL8 enhanced TNF $\alpha$ - or IL-1 $\beta$ induced NF- $\kappa$ B activation (Fig. $2 b$ and Supplementary Fig. 2a), and the level of NF- $\mathrm{BB}$ activation was correlated with the knockdown efficiency. As a negative control experiment, ANGPTL8-RNAi did not affect the IFN $\gamma$-induced IRF1 activation, which is another pathway involved in immunity and separated from $\mathrm{TNF} \alpha$-mediated signaling (Fig. 2c). These data indicate that ANGPTL8 specifically potentiates the TNF $\alpha$-triggered and IL-1 $\beta$-triggered NF- $\kappa \mathrm{B}$ activation.

Further quantitative real-time PCR (qPCR) analysis demonstrated that knockdown of ANGPTL8 significantly potentiated the TNF $\alpha$-triggered transcription of NF- $\mathrm{KB}$ downstream genes, such as IL8, CXCL2, and NFKBIA (Fig. 2d). ANGPTL8-RNAi-\#3 was used in following experiments for its highest efficiency. Similar to HepG2 cells, knockdown of ANGPTL8 also potentiated the TNF $\alpha$ - or IL-1 $\beta$-triggered NF- $\kappa \mathrm{B}$ activation in HEK293T and A549 cells (Supplementary Fig. 2b, c). Consistently, knockdown of ANGPTL8 enhanced TNF $\alpha$-induced phosphorylation of IKKs and $\mathrm{I} \kappa \mathrm{B} \alpha$, two hallmarks of NF- $\kappa \mathrm{B}$ activation (Fig. 2e).

To confirm the role of ANGPTL8 in TNF $\alpha$-mediated NF- $\kappa B$ activation, we generated ANGPTL8-deficient HepG2 cell lines by using the CRISPR-Cas9 system. The ANGPTL8-deficient $\left(A N G P T L 8^{-/-}\right)$clones were confirmed at DNA and protein levels (Fig. 3a, b). In reporter assays, the ANGPTL $8^{-1-}$ cells showed significantly enhanced NF- $\mathrm{KB}$ activation comparing to the wild-type cells after TNF $\alpha$ or IL-1 $\beta$ induction (Fig. $3 c$ and

a

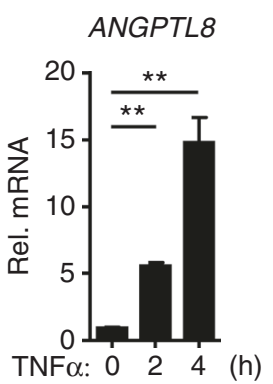

b

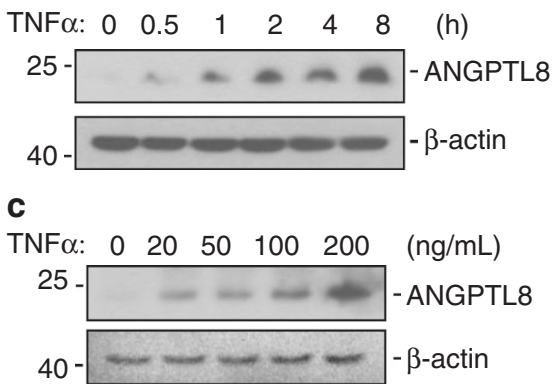

Fig. 1 TNF $\alpha$ upregulates the expression of ANGPTL8 in HepG2 cells. a, b The transcription level $(\mathbf{a}, n=3)$ and protein level (b) of ANGPTL8 after $\mathrm{TNF} \alpha(50 \mathrm{ng} / \mathrm{mL})$ treatment. The following experiments used the same dose of TNF $\alpha$ if not mentioned. $\mathbf{c}$ The protein level of ANGPTL8 at $8 \mathrm{~h}$ after different dosages of TNF $\alpha$. Data are shown as the mean \pm SEM, unpaired two-tailed student's test was used for statistics (a), ${ }^{\star \star} p<0.01$. Data are representative of three independent experiments 
a

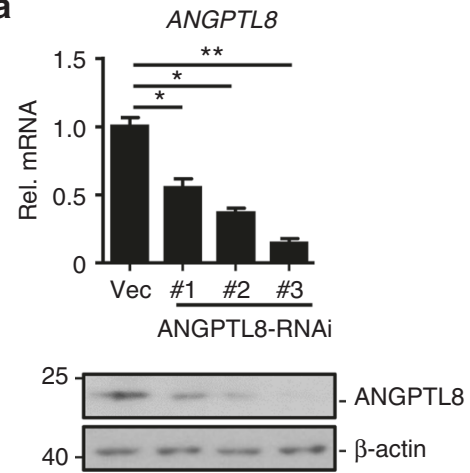

b

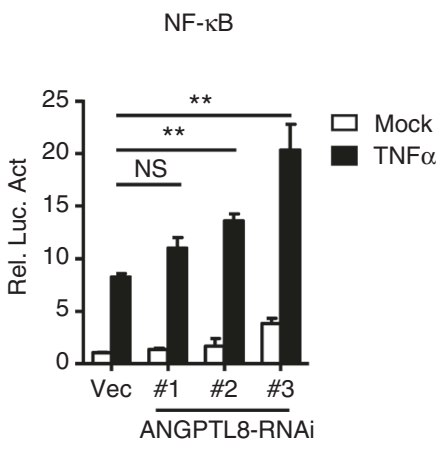

C

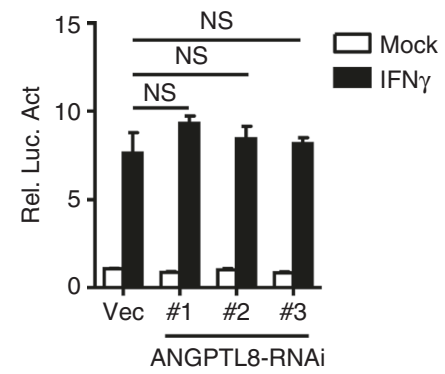

d

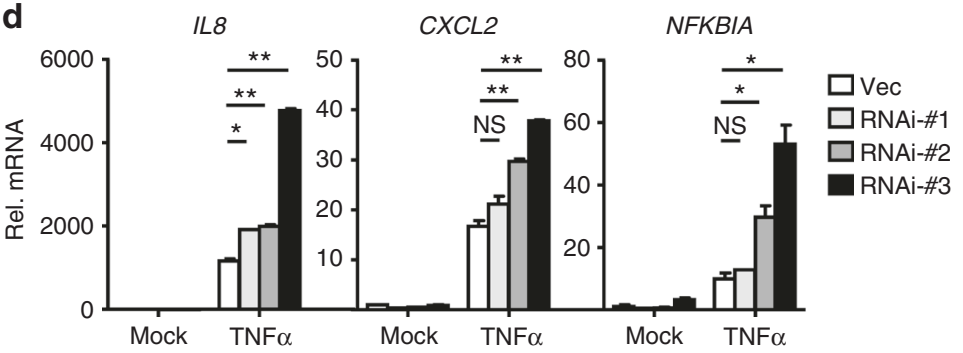

e

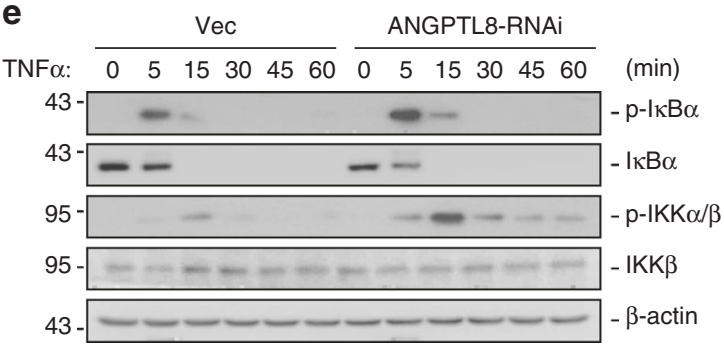

Fig. 2 Knockdown of ANGPTL8 potentiates TNF $\alpha$-induced NF- $\kappa B$ activation. a Efficacy of three different ANGPTL8-RNAi plasmids on the transcription (upper panels) and expression (lower panels) of ANGPTL8 in the control or shANGPTL8 stable HepG2 cell lines $(n=3)$. b Effects of ANGPTL8-RNAi on TNF $\alpha$-induced NF-KB activation in the cell lines indicated as in $\mathbf{a}(n=3)$. $\mathbf{c}$ Effects of ANGPTL8-RNAi on IFN $\gamma(100 \mathrm{ng} / \mathrm{mL})$ induced IRF1 activation $(n=3)$. d Effects of ANGPTL8-RNAi on TNF $\alpha$-induced IL8, CXCL2 and NFKBIA transcription for $2 \mathrm{~h}$ in cell lines indicated as in a $(n=3)$. e Effects of ANGPTL8-RNAi on TNF $\alpha$-induced IKK and IKB $\alpha$ phosphorylation in ANGPTL8-RNAi-\#3 or control cells in a. Data are shown as the mean \pm SEM, unpaired two-tailed Student's test was used for statistics (a-d), ${ }^{\star} p<0.05,{ }^{\star \star} p<0.01, N S>0.05$. Data are representative of three independent experiments

Supplementary Fig. 2d). Knockout of ANGPTL8 facilitated TNF $\alpha$-induced IL8, CXCL2 and TNFA transcription (Fig. 3d). In contrast, the level of IFN $\gamma$-induced STAT1 transcription was comparable between the wild-type and ANGPTL $8^{-1-}$ cells (Fig. 3e). We next reconstituted the ANGPTL8 knockout cells with Flag-tagged ANGPTL8 by retrovirus-mediated gene transfer (Fig. 3f), and found that reconstitution of ANGPTL8 into ANGPTL $8^{-1-}$ cells suppressed the TNF $\alpha$-triggered IL8, CXCL2 and TNFA transcriptions (Fig. 3g). Collectively, we demonstrate that endogenous ANGPTL8 negatively regulates TNF $\alpha$-triggered $\mathrm{NF}-\kappa \mathrm{B}$ signaling.

ANGPTL8 regulates NF-кB activation at the IKK complex level. We next investigated the molecular mechanisms by which ANGPTL8 regulates the TNF $\alpha$ or IL- $1 \beta$ signaling mediated NF$\kappa \mathrm{B}$ activation. TNF $\alpha / \mathrm{IL}-1 \beta$-mediated NF- $\mathrm{KB}$ activation includes three major steps: adaptors such as TRAF2/6-mediated or RIP1mediated recruitment of IKK complex to TNFR, activation of IKK $\beta$, and activation of NF- $\kappa B$. We cotransfected HEK293T cells with a vector expressing TRAF2, TRAF6, RIP1, IKK $\beta$, or p65, together with an overexpression or knockdown vector of ANGPTL8 plus an NF- $\mathrm{kB}$ luciferase reporter vector. The overexpression/knockdown of ANGPTL8 induced changes of NF- $\mathrm{BB}$ activation upon overexpression of TRAF2/6 or RIP1, but not IKK $\beta$ and p65 (Fig. 4a, b). These data implicate that ANGPTL8 may involve in the recruitment or activation of IKK complex.

Consistently, by co-IP experiments, we found that ANGPTL8 interacted with RIP1, IKK $\beta$ and IKK $\gamma$ in the cells cooverexpressed ANGPTL8 and the regulator molecules involved in NF- $\kappa$ B activation cascade (Fig. 4c). Moreover, in untransfected cells, ANGPTL8 rapidly interacted with IKK $\beta / \gamma$ and RIP1 upon TNF $\alpha$ treatment (Fig. $4 \mathrm{~d}$ ), which may in part due to the TNF $\alpha$ triggered ANGPTL8 expression. Collectively, these results indicate that ANGPTL8 may target IKK complex through participating in the recruitment or activation of IKKs.

Since ANGPTL8 is mostly known as a secreted protein, after we found its role in the regulation of intracellular signaling, we studied the localization of ANGPTL8. In ANGPTL8 overexpression HepG2 cells, a large amount of ANGPTL8 was detected in cell lysate, while a proportion of ANGPTL8 was secreted (Supplementary Fig. 3a), this result was consistent with a recent study carried out in HEK293T cells ${ }^{23}$. Immunofluorescence experiments confirmed that endogenous ANGPTL8 had intracellular localization (Supplementary Fig. 3b).

ANGPTL8 facilitates the degradation of IKK $\boldsymbol{\gamma}$. We next investigated how ANGPTL8 regulates the IKK complex. Interestingly, we found that overexpression of ANGPTL8 resulted in markedly decreased expression level of Flag-IKK $\gamma$, but not FlagIKK $\alpha$, -IKK $\beta$, -TRAF6, or -RIP1 (Fig. 5a), whereas knockdown of ANGPTL8 showed the opposite effects (Fig. 5b). Furthermore, knockdown of ANGPTL8 attenuated TNF $\alpha$-induced degradation of IKK $\gamma$ but not that of IKK $\alpha / \beta$ (Fig. 5c), without affecting the transcription of IKK $\gamma$ (Supplementary Fig. 4). These data suggested that ANGPTL8 promotes the degradation of IKK $\gamma$. Besides, knockdown or knockout of ANGPTL8 significantly potentiated the TNF $\alpha$-induced transcription of IL8 and CXCL2, which was abolished by further knockdown of IKK $\gamma$ (Fig. 5d, e), suggesting that ANGPTL8 facilitates TNF $\alpha$-induced NF- $\mathrm{KB}$ activation by degrading IKK $\gamma$.

ANGPTL8 mediates autophagic IKK $\boldsymbol{\gamma}$ degradation. Protein degradation is one of the key ways to turn off signaling transduction. Proteins in eukaryotes can be degraded by UPS or autophagy. ANGPTL8-mediated IKK $\gamma$ degradation was completely blocked by 3-methyladenine (3MA) and chloroquine (CQ), inhibitors for autophagosome and lysosome, respectively; 
a

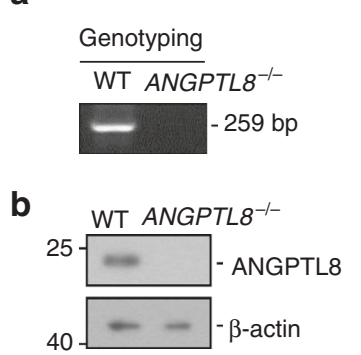

C

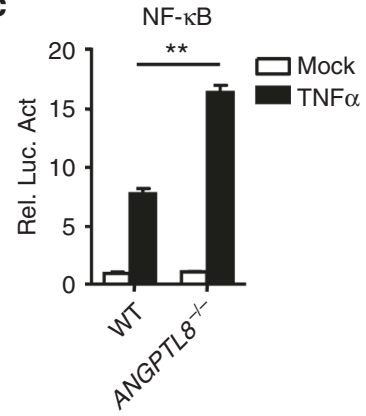

d

d

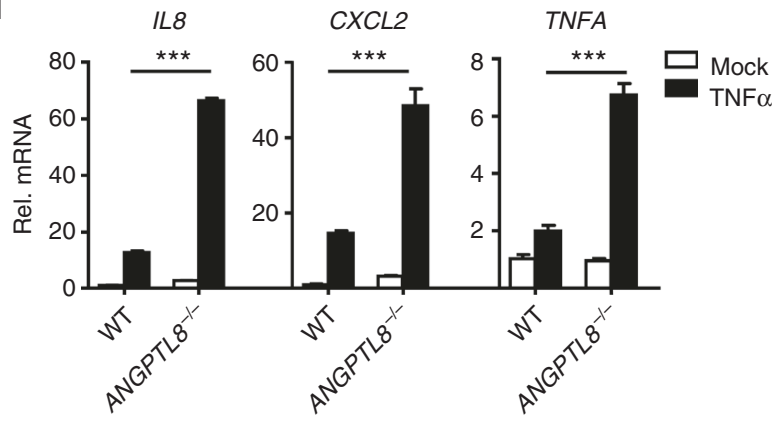

f

e

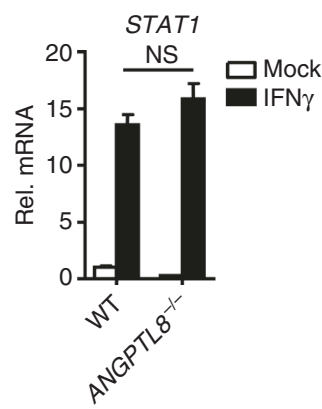

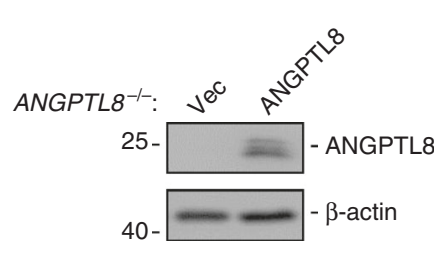

g

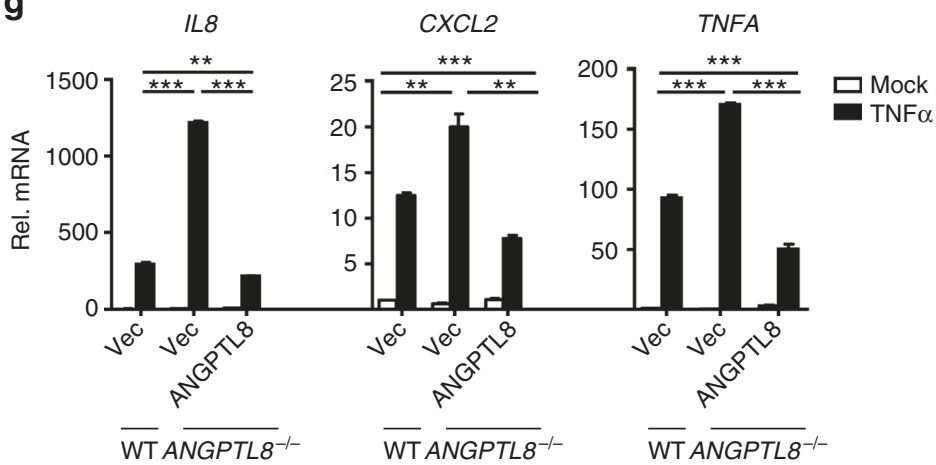

Fig. 3 Knockout of ANGPTL8 potentiates TNF $\alpha$-induced NF- $\kappa B$ activation. a Genotyping of ANGPTL8 ${ }^{-/-}$and wild-type HepG2 cells. b The expression level of ANGPTL8 in ANGPTL8 ${ }^{-/}$and wild-type HepG2 cells. $\mathbf{c}$ Effects of ANGPTL8 deficiency on TNF $\alpha$-induced NF-kB activation in luciferase reporter assays $(n=3)$. $\mathbf{d}$ Effects of ANGPTL8 deficiency on TNF $\alpha$-induced IL8, CXCL2 and TNFA transcription $(n=3)$. e Effects of ANGPTL8 deficiency on IFN $\gamma$-induced STAT1 transcription $(n=3)$. $\mathbf{f}$ Reconstitution of ANGPTL8-Flag in the ANGPTL8 ${ }^{-/-}$cell lines. $\mathbf{g}$ Effects of ANGPTL8 deficiency and reconstitution on TNF $\alpha$-induced IL8,CXCL2, and TNFA transcription $(n=3)$. Data are shown as the mean \pm SEM, unpaired two-tailed student's test was used for statistics $(\mathbf{c}, \mathbf{d}, \mathbf{e}, \mathbf{g}),{ }^{\star \star} p<0.01,{ }^{\star \star \star} p<0.001, \mathrm{NS}>0.05$. Data are representative of three independent experiments

whereas the proteasome inhibitor MG132 could not inhibit the ANGPTL8-mediated IKK $\gamma$ degradation (Fig. 6a). Autophagyrelated 5 (ATG5) and ATG7 are essential adaptors for the autophagic degradation, and knockdown of ATG5/7 inhibited the rapamycin-induced turnover of LC3 (Supplementary Fig. 5) $24,25$. Consistently, ANGPTL8-mediated IKK $\gamma$ degradation was dramatically attenuated in ATG5-/ATG7-RNAi cells (Fig. 6b). These results suggest ANGPTL8 mediates IKK $\gamma$ degradation in a macroautophagy-dependent manner.

Confocal microscopy experiments further suggested that in unstimulated cells, only a small fraction of IKK $\gamma$ was co-localized with GFP-LC3 dots, a marker for autophagosome; whereas in cells overexpressing ANGPTL8, the overlap and correlation efficiency between IKK $\gamma$ and GFP-LC3 dots were markedly increased (Fig. 6c, d). It has been observed that TNF $\alpha$ stimulation induces relocalization of IKK $\gamma$ into punctate structures that are enriched in activated IKK kinases and IKK $\gamma$, which may be essential for NF- $\kappa B$ activation ${ }^{26,27}$. Consistently, we found that TNF $\alpha$ stimulation induces the recruitment of IKK $\gamma$ into punctate structures, however, this TNF $\alpha$-induced IKK $\gamma$ punctual relocalization was dramatically decreased in ANGPTL $8^{-/-}$cells, and the co-localization of IKK $\gamma$ with GFP-LC3 was also significantly reduced (Fig. 6e, f).

ANGPTL8 and p62 co-mediate the autophagic degradation of IKK $\boldsymbol{\gamma}$. Motivated by the observations that ANGPTL8 selectively mediated the autophagic degradation of IKK $\gamma$ but not IKK $\alpha / \beta$, we next studied whether ANGPTL8 mediates IKK $\gamma$ degradation via selective autophagy. K63-linked ubiquitin chains have been reported to promote selective autophagy-dependent degradation for specific target proteins ${ }^{14}$, we found overexpression of wildtype or K63-linked ubiquitin (the ubiquitin in which all of the lysine mutated to arginine except the lysine 63) enhanced the ANGPTL8-mediated IKK $\gamma$ degradation (Fig. 7a and Supplementary Fig. 6); whereas CYLD, which removes the K63-linked ubiquitin chains of $\mathrm{IKK} \gamma^{28}$, attenuated ANGPTL8-mediated IKK $\gamma$ degradation in a dose-dependent manner (Fig. 7b).

Typically, cargoes are selectively transferred by one or more of the known autophagy receptors (p62, Tollip, NDP52, NBR1 and OPTN etc.) for degradation ${ }^{15,29}$. We found that it was p62 and Tollip, but not NDP52, NBR1 or OPTN, that mediated the degradation of IKK $\gamma$, which was further markedly enhanced when co-expressed with ANGPTL8 (Fig. 7c and Supplementary Fig. 7a). In wild-type cells, although p62 and Tollip both significantly and dose-dependently induced IKK $\gamma$ degradation, ANGPTL8 deficiency only reversed p62-mediated, but not Tollip-mediated degradation of IKK $\gamma$ (Fig. $7 \mathrm{~d}$ and Supplementary Fig. 7b). Moreover, ANGPTL8 could interact with p62, but not Tollip; and the interaction between ANGPTL8 and p62 was greatly enhanced after TNF $\alpha$ treatment (Fig. 7e). Also, knockdown of p62 dramatically attenuated ANGPTL8-mediated IKK $\gamma$ degradation (Fig. 7f). The LC3 interacting region (LIR) of p62 is responsible for recruitment of LC3 to the autophagosome ${ }^{30}$. Here, we observed that p62 LLIR (a LIR domain deleted p62 mutant) showed dramatically decreased ability in mediating IKK $\gamma$ degradation (Supplementary Fig. 7c). Notably, similar to p62 and IKK $\gamma$, we found that ANGPTL8 also underwent autophagic degradation (Supplementary Fig. 7d). Altogether, these data indicate that ANGPTL8 and p62 work collaboratively to mediate the autophagic degradation of IKK $\gamma$.

Reducing ANGPTL8 attenuates the IKK $\gamma-\mathrm{p} 62$ interaction. In selective autophagy, substrate recognition by autophagy receptor is essential for cargo selection. By performing co-IP experiments 
a

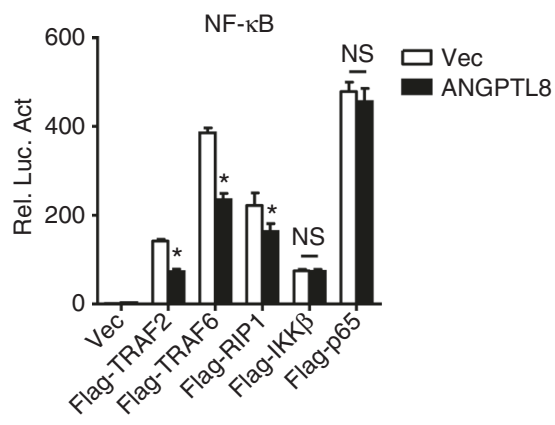

b

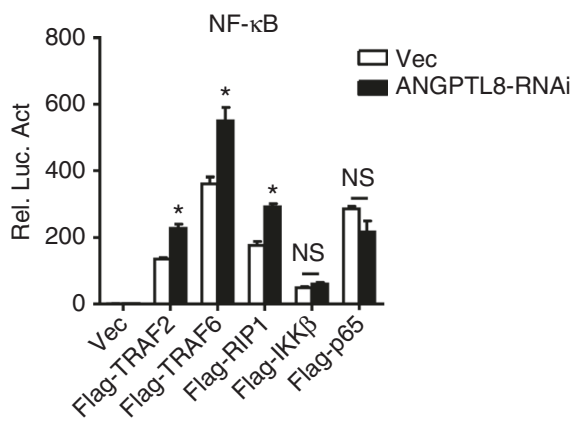

C

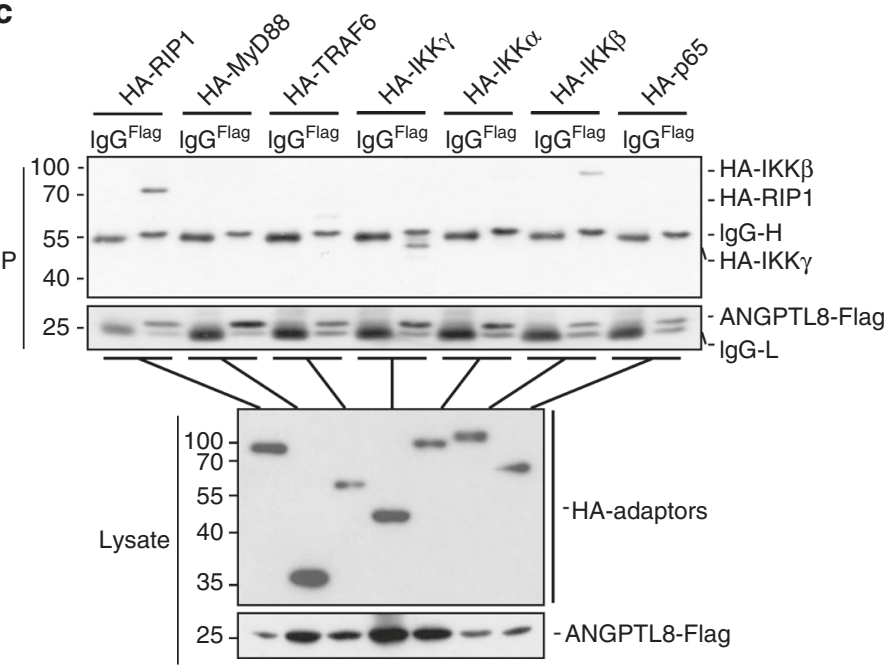

d

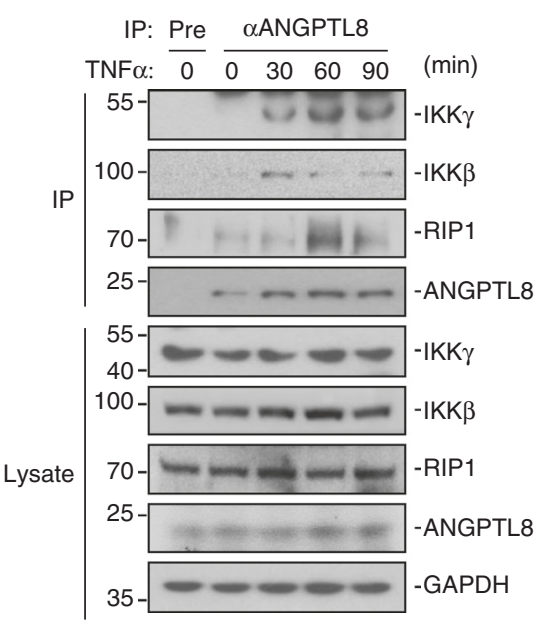

Fig. 4 ANGPTL8 regulates TNF $\alpha$-induced NF-KB activation at the IKK complex level. a Effects of the ANGPTL8 overexpression on Flag-TRAF2-, FlagTRAF6-, Flag-RIP1-, Flag-IKK $\beta$ - or Flag-p65-mediated NF-кB activation. HEK293T cells were transfected with a control vector or ANGPTL8-Flag expression plasmid $(0.05 \mu \mathrm{g})$ and the indicated plasmids $(0.1 \mu \mathrm{g}$ of TRAF2 or IKK $\beta, 0.01 \mu \mathrm{g}$ of TRAF6, RIP1 or p65). Reporter assays were performed $20 \mathrm{~h}$ after transfection $(n=3)$. $\mathbf{b}$ Effects of the ANGPTL8-RNAi on Flag-TRAF2-, Flag-TRAF6-, Flag-RIP1-, Flag-IKK $\beta$ - or Flag-p65-mediated NF- $\mathrm{kB}$ activation. HEK293T cells were transfected with a control vector or ANGPTL8-RNAi-\#3 plasmid $(0.3 \mu \mathrm{g})$ for $24 \mathrm{~h}$ before being transfected with the indicated plasmids. Twenty hours later, the cells were lysed and reporter assays were performed $(n=3)$. c ANGPTL8 interacts with IKK $\gamma$, IKK $\beta$ and RIP1 in the mammalian overexpression system. $\mathbf{d}$ Endogenous ANGPTL8 interacts with IKK $\gamma$, IKK $\beta$ and RIP1 in HepG2 cells. Data are shown as the mean \pm SEM, unpaired two-tailed student's test was used for statistics $(\mathbf{a}, \mathbf{b}),{ }^{\star} p<0.05, \mathrm{NS}>0.05$. Data are representative of three independent experiments

in physiological conditions, we found that IKK $\gamma$, ANGPTL8 and p62 interacted with each other (Fig. 8a, b). We noted that only a small fraction of p62 was associated with IKK $\gamma$ in resting cells, and this association was greatly increased after $\mathrm{TNF} \alpha$ treatment, which is consistent with the observation that ANGPTL8 was simultaneously recruited to IKK $\gamma$ (Fig. 8a, b). Consistently, TNF $\alpha$-induced interaction between IKK $\gamma$ and p62 was impaired by knockdown or knockout of ANGPTL8 (Fig. 8c, d). These data indicate that upon TNF $\alpha$ stimulation, IKK $\gamma, \mathrm{p} 62$, and ANGPTL8 form a complex in which ANGPTL8 plays an important role in mediating the interaction.

Oligomerization of ANGPTL8 is essential for IKK $\gamma$ degradation. In selective autophagy, protein aggregation is a key event in mediating cargo selection and separation ${ }^{31}$. Interestingly, by coIP analysis, we found ANGPTL8 can self-oligomerize (Fig. 9a). ANGPTL8 is predicted to have an N-terminal signal peptide (residues 1-25) and two coiled-coil (CC) domains (residues 77-134 and 156-193) which are presumably associated with protein-protein interaction (Fig. 9b); however, the function of different domains/regions in ANGPTL8 is not clear ${ }^{32,33}$. By using domain mapping, we identified the region between residues 26-70 as an essential domain for the self-oligomerization of ANGPTL8 (Fig. 9a). Notably, the truncation mutants (71-C and
$\Delta 26-70)$ that lost the self-association capacity could not mediate the IKK $\gamma$ degradation, while the CC domain that unrelated to the self-oligomerization of ANGPTL8 was dispensable in this process (Fig. 9c and Supplementary Fig. 8). Consistently, ANGPTL8$\Delta 26-70$ showed a significantly diminished interaction with IKK $\gamma$ and lost the ability of inhibiting TNF $\alpha$-induced NF- $\kappa$ B activation (Fig. 9d, e). Thus, oligomerization of ANGPTL8 mediated by its $\mathrm{N}$-terminal 26-70 region is essential for the ANGPTL8-mediated inhibition of NF- $\kappa \mathrm{B}$ activation by mediating the interaction and degradation of IKK $\gamma$. Moreover, we found that the coiled-coil domains were responsible for the ANGPTL8-p62 interaction (Supplementary Fig. 9). Interestingly, compared with the fulllength ANGPTL8, ANGPTL8- $\Delta 26-70$ showed a stronger interaction with p62, suggesting the self-oligomerization is not required for ANGPTL8-p62 interaction (Fig. 9d).

Gel filtration analysis was performed to explore the oligomerization status of ANGPTL8. The full-length ANGPTL8 was most abundant in a higher molecular mass (fractions 5-12) than ANGPTL8- $\Delta 26-70$ (fractions 10-15) (Fig. 9f). Transfection of ANGPTL8- $\Delta 26-70$ led to significantly decreased molecular weight of p62 and IKK $\gamma$-containing fractions in the same experiments, indicating the importance of ANGPTL8 oligomerization in the formation of IKK $\gamma$ and p62-containing protein complex. We also expressed and purified the recombinant ANGPTL8 to assess its oligomerization tendency in vitro 


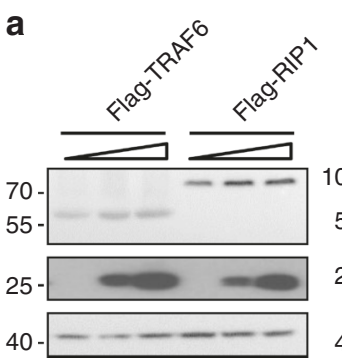

C

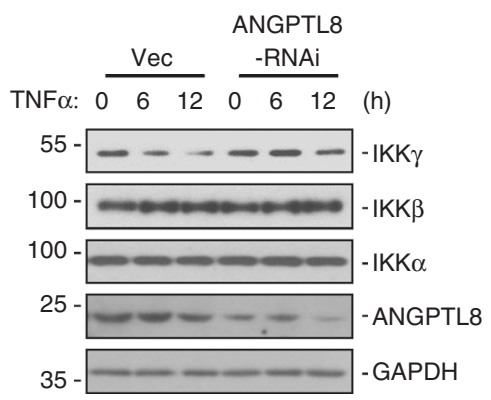

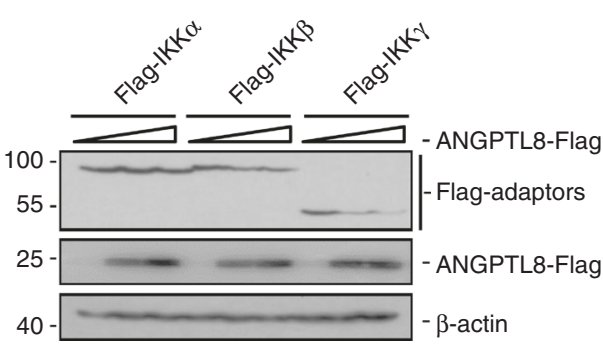

d

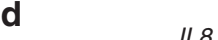

b

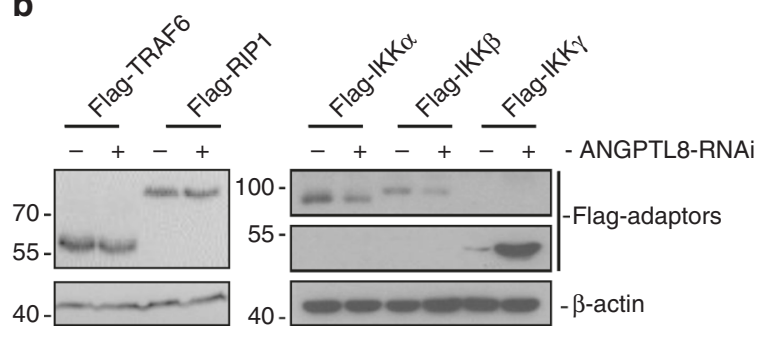

e
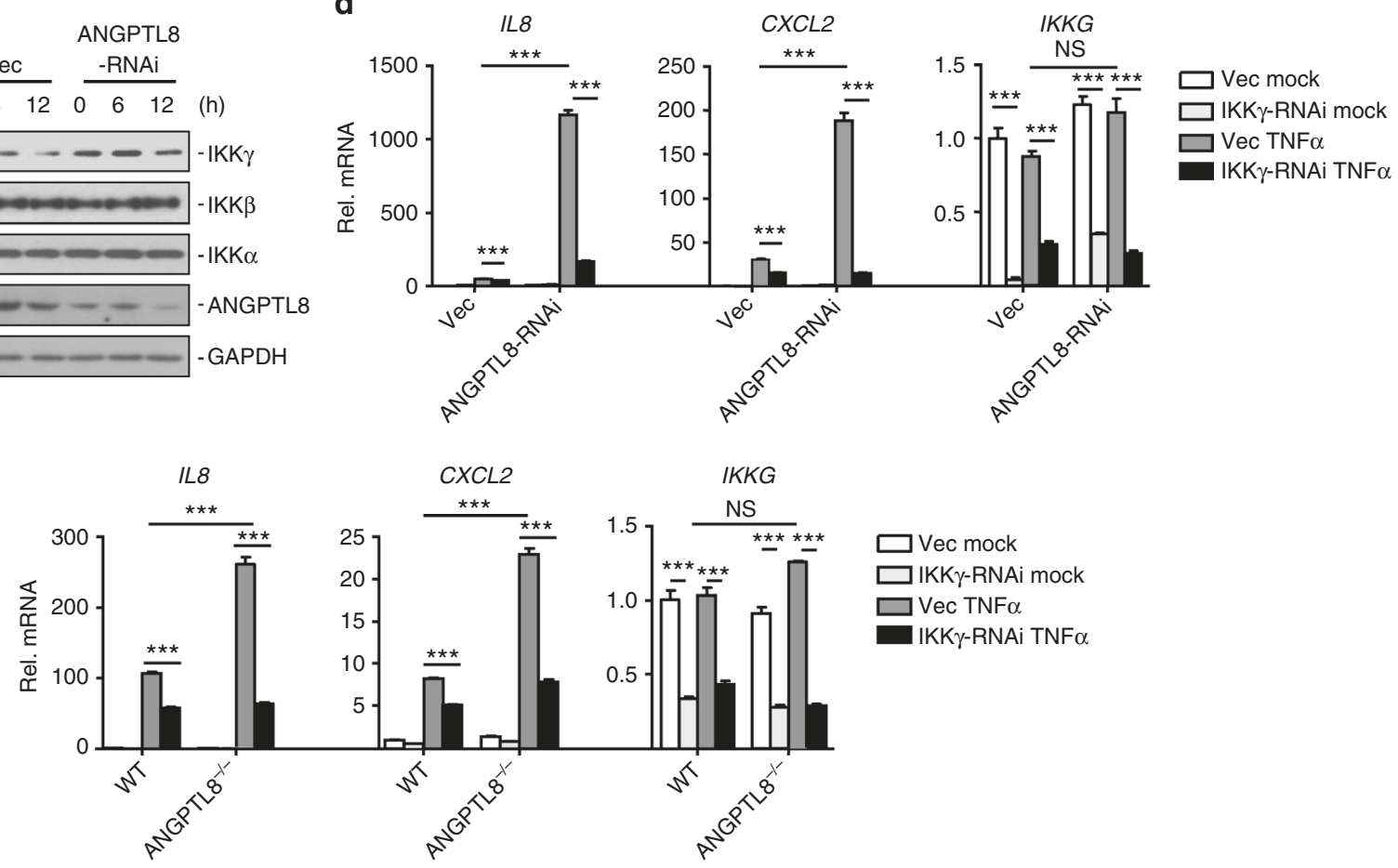

Fig. 5 ANGPTL8 facilitates the degradation of IKK $\gamma$. a Effects of ANGPTL8 overexpression on the expression level of transfected TRAF6, RIP1, IKK $\alpha$, IKK $\beta$ or IKK $\gamma$ in HEK293T cells. b Effects of ANGPTL8-RNAi on the expression level of transfected TRAF6, RIP1, IKK $\alpha$, IKK $\beta$, or IKK $\gamma$. c Effects of ANGPTL8-RNAi on the expression levels of the endogenous IKK $\alpha, I K K \beta$ or IKK $\gamma$ after TNF $\alpha$ and $\mathrm{CHX}$ co-treatment. The indicated cells were treated by $\mathrm{CHX}$ (200 $\mu \mathrm{g} / \mathrm{mL}$ ) for $1 \mathrm{~h}$, followed by TNF $\alpha$ treatment for the indicated time. $\mathbf{d}$ Knockdown of IKK $\gamma$ inhibits ANGPTL8-RNAi facilitated NF- $\kappa B$ activation triggered by TNF $\alpha$ (2 h) in the ANGPTL8-RNAi or control cell lines $(n=3)$. e Knockdown of IKK $\gamma$ inhibits ANGPTL8 deficiency-facilitated NF- $\kappa B$ activation triggered by TNF $\alpha$ for $2 \mathrm{~h}$ $(n=3)$. Data are shown as the mean \pm SEM, unpaired two-tailed student's test was used for statistics (d, e). ${ }^{\star \star \star} p<0.001$, NS $>0.05$. Data are representative of three independent experiments

(Supplementary Fig. 10a). The circular dichroism spectra revealed that in solution, ANGPTL8 adapted a mixed $\alpha$-helix/ $\beta$-structures/ random coil structure (Supplementary Fig. 10b, c), which agrees with the structural prediction (Supplementary Fig. 10d). ANGPTL8 showed tendency to aggregate into oligomers and large aggregates (fibrils) as measured by the dynamic light scattering (DLS) assays or by probing with the anti-oligomer and anti-fibril antibodies (Supplementary Fig. 10e, f). Under transmission electronic microscopy, oligomers and fibrils were also identified (Supplementary Fig. 10g).

Potential involvement of ANGPTL8 in acute inflammation. Our in vitro studies suggest that ANGPTL8 degrades IKK $\gamma$, whose tight regulation is essential for the balance of inflammation, we next investigated the physiological relevance of ANGPTL8 in vivo. As demonstrated by qPCR analysis (Supplementary Fig. 11A), Angptls is relatively high in the liver and brown adipose tissue (BAT), and low in the spleen, lung and kidney of mouse. Next, we examined the tissue level of Angptls in mice challenged with lipopolysaccharide (LPS), a constituent of the Gram-negative bacteria outer membranes and an important microbial trigger that stimulates innate immunity. Interestingly, in tissues with high level of Angptl8, fast upregulation and downregulation of Tnfa transcription during the acute phase $(0-1 \mathrm{~h})$ and the resolution phase $(1-6 \mathrm{~h})$ was respectively observed upon LPS challenge; in contrast, they were relatively slow in tissues with low Angptl8 expression (Fig. 10a and Supplementary Fig. 11b). This observation implicates that for tissues that are sensitive to the inflammatory stress, larger amount of "brake" molecules, such as ANGPTL8, may be demanded. Furthermore, upon LPS stress, the expression of Angptl8 was increased, while Ikk $\gamma$ expression was decreased (Fig. 10b, c); however, the interaction among Ikk $\gamma$, p62 and Angptl8 was enhanced (Fig. 10d). These results are consistent with our in vitro experiments.

Third, we measured the circulating ANGPTL8 level in the blood of two groups of patients with systemic inflammatory response syndrome. One group includes patients with positive detection of procalcitonin (PCT) which is a biomarker for early 
a

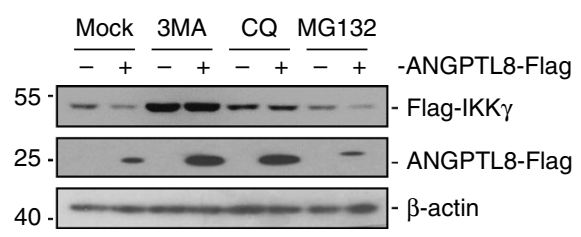

b
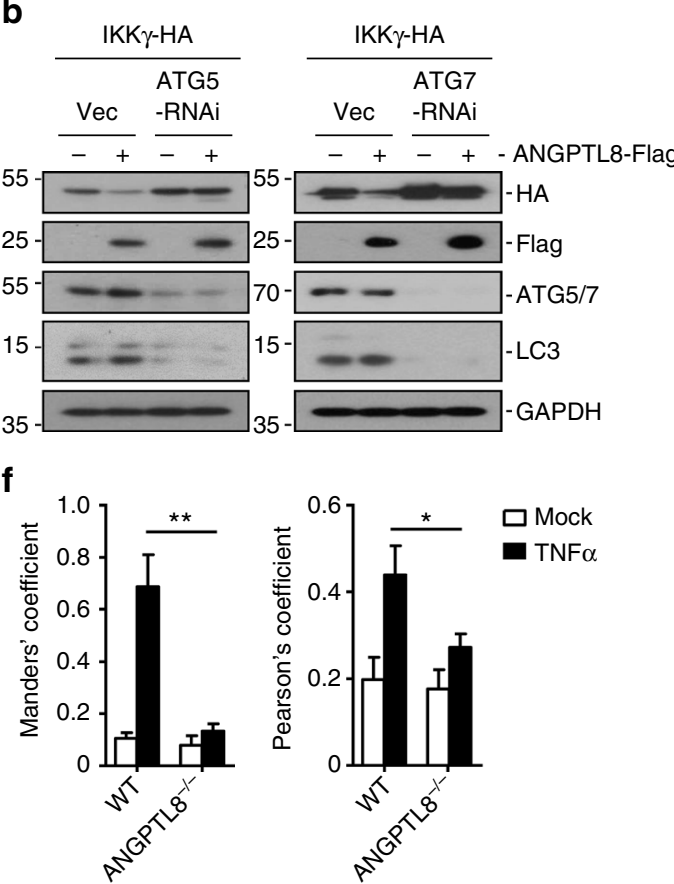

C

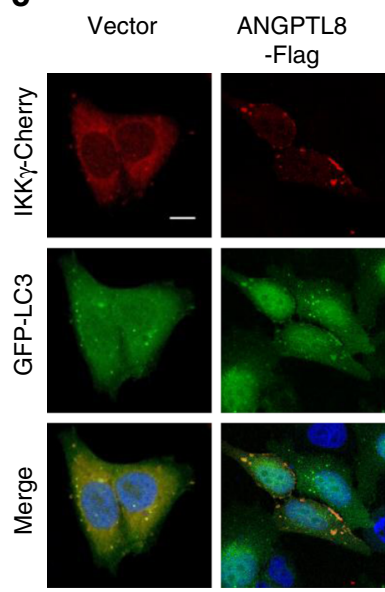

e

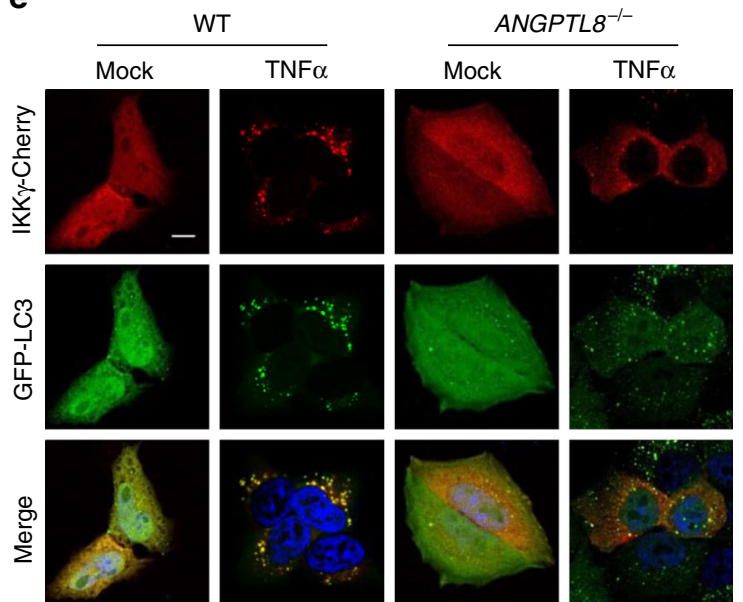

Fig. 6 ANGPTL8 mediates the autophagic IKK $\gamma$ degradation. a Effects of 3MA, CQ, and MG132 on ANGPTL8-mediated destabilization of IKK $\gamma$. HEK293T cells $\left(1 \times 10^{5}\right)$ were transfected with the indicated plasmids. Fourteen hours after transfection, the cells were treated with the indicated inhibitors (3MA, $3.35 \mathrm{mM}$; CQ, $0.05 \mathrm{mM} ; \mathrm{MG132}, 10 \mu \mathrm{M}$ ) for $6 \mathrm{~h}$ before immunoblots. b Effects of the knockdown of ATG5/7 on ANGPTL8-mediated IKK $\gamma$ proteolysis. The HEK293T was transfected with ATG5/7-RNAi for $12 \mathrm{~h}$ and followed by puromycin selection $(1 \mu \mathrm{g} / \mathrm{mL})$ for $12 \mathrm{~h}$ before the indicated plasmids were transfected for $24 \mathrm{~h}$, and then cells were collected for immunoblots. c, $\mathbf{d}$ The representative images (c) of and quantitative results (d) of effects of ANGPTL8 on the translocation of IKK $\gamma$ to autophagosomes, the scale bar represents $10 \mu \mathrm{m}(n=6)$. e, $\mathbf{f}$ The representative images $(\mathbf{e})$ and quantitative results (f) of effects of ANGPTL8 deficiency on TNF $\alpha$-induced co-localization of IKK $\gamma$ with the autophagosomes. The scale bar represents 10 $\mu \mathrm{m}(n=6)$. Data are shown as the mean \pm SEM, unpaired two-tailed student's test was used for statistics $(\mathbf{d}, \mathbf{f}) .{ }^{\star} p<0.05,{ }^{\star \star} p<0.001$. Data are representative of three independent experiments

diagnosis of sepsis $^{27}$, the other group includes patients with positive detection of endotoxin which is an important microbiological assessment for Gram-negative bacteria-mediated inflammation ${ }^{34}$. Compared to the healthy subjects, the circulating ANGPTL8 level was dramatically increased in both groups of patients (Fig. 10e and Supplementary Table 1). Collectively, these results indicated that ANGPTL8 can be induced by inflammatory stimuli in mouse and human, and may thus play roles in shutting down acute inflammatory response.

\section{Discussion}

ANGPTL8 has been known as a potent regulator of lipid metabolism ${ }^{35}$. Circulating ANGPTL8 is increased in patients with T2D or non-alcoholic fatty liver diseases, which makes ANGPTL8 an attractive therapeutic target for metabolic syndromes ${ }^{22,36,37}$. On the other hand, inflammation, especially the proinflammatory cytokines-mediated chronic inflammation, has been demonstrated to contribute to the development of metabolic diseases such as T2D. However, the role of ANGPTL8 in inflammation is unknown. As a central event of inflammation and immunity, TNF $\alpha$-induced $N F-\kappa B$ activation must be tightly controlled to avoid inflammatory diseases, autoimmunity and cancers $^{6-8}$. Here we present the first evidence that multiple inflammatory stimuli, including $\mathrm{TNF} \alpha$, induce the transcription and expression of ANGPTL8 in vitro and in vivo; the latter then forms a protein complex with p62 and IKK $\gamma$, in which ANGPTL8 works as a co-receptor of p62 and facilitates the autophagic IKK $\gamma$ degradation, thereby inhibiting the TNF $\alpha$-induced NF- $\mathrm{KB}$ activation (working model shown in Fig. 10f). The ANGPTL8/p62$\mathrm{IKK} \gamma$ axis thus serves as a negative feedback loop to restrict the TNF $\alpha$-trigged NF- $\kappa$ B activation and inflammation.

Since ANGPTL8 is mostly known as a secreted protein, it is interesting that our results suggest it also plays intracellular roles and has intracellular location. Actually, other studies have demonstrated that some secreted proteins have intracellular functions. For example, ISG15, an interferon-induced modifier, is found both intracellularly and extracellularly; the secreted ISG15 has cytokine like activities ${ }^{38}$; whereas the intracellular ISG15 can conjugate various proteins via ISGylation, and prevents the IFN- 
a

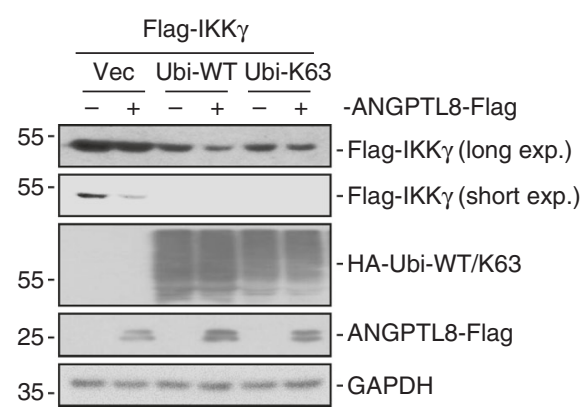

C

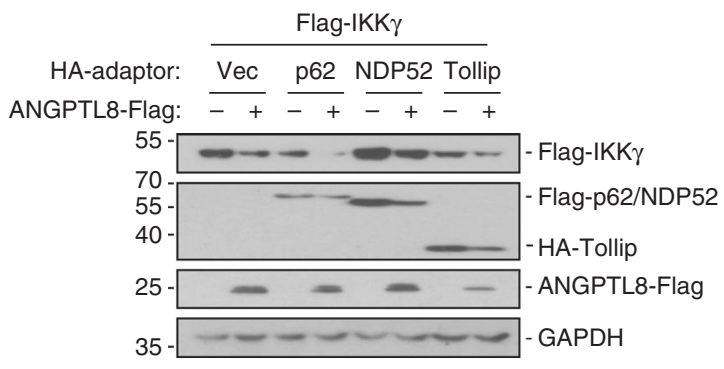

d
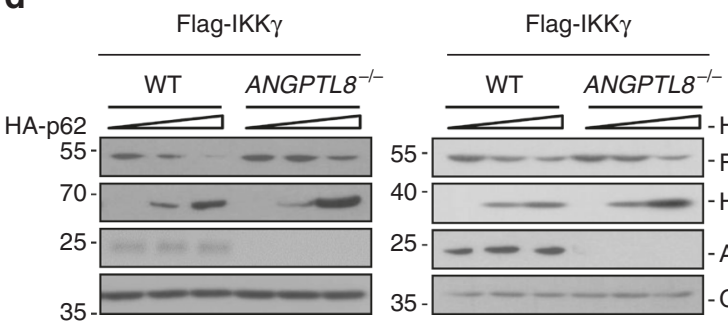

b

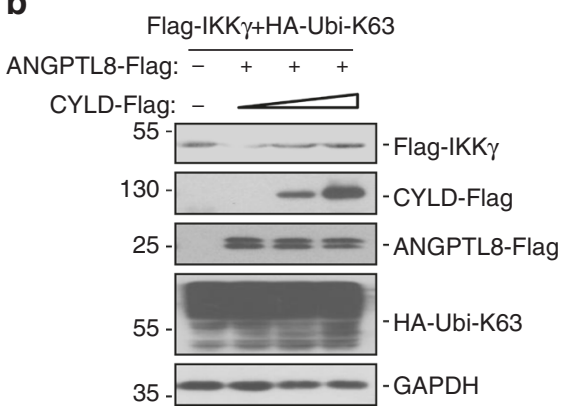

e

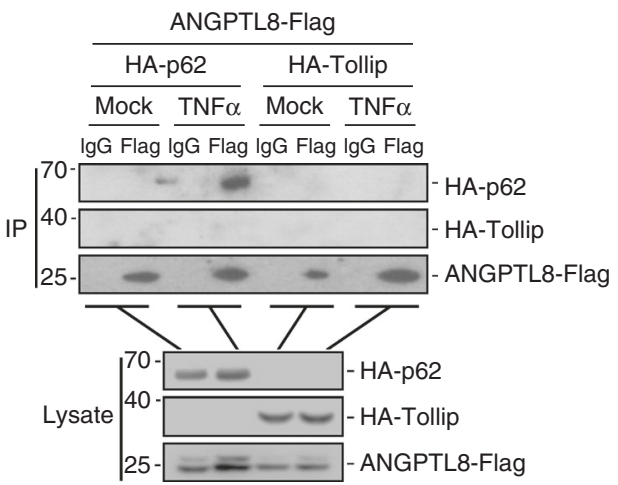

f

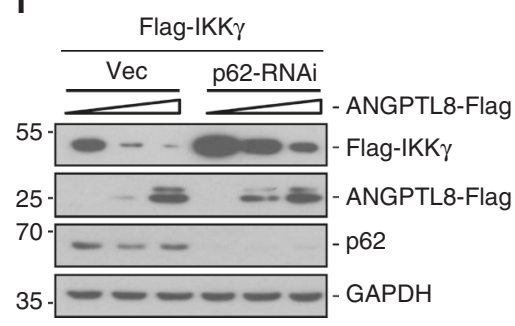

Fig. 7 ANGPTL8 and p62 collaboratively mediate the autophagic degradation of IKK $\gamma$. a The effects of ubiquitin on the ANGPTL8-mediated IKK $\gamma$ degradation in HEK293T cells. Ubi-WT, the wild-type ubiquitin; Ubi-K63, the ubiquitin in which all of lysine (K) mutated to arginine (R) except K63. $\mathbf{b}$ The effect of deubiquitinase CYLD on the ANGPTL8-mediated IKK $\gamma$ degradation. $\mathbf{c}$ The effects of different autophagic adaptors on the ANGPTL8-mediated IKK $\gamma$ degradation. $\mathbf{d}$ Overexpression of $\mathrm{p} 62$ but not Tollip-mediated IKK $\gamma$ degradation is attenuated in ANGPTL8 ${ }^{-/-}$cells. e ANGPTL8 interacts with p62 but not Tollip in the mammalian overexpression system. f ANGPTL8-mediated IKK $\gamma$ degradation is decreased in p62-RNAi stable cell line. Data are representative of three independent experiments

$\alpha / \beta$-dependent autoinflammation ${ }^{39}$. PCSK9 is another secreted protein, and binds hepatotic LDLRs both extracellularly and intracellularly which leads to LDLRs degradation ${ }^{40,41}$. Similarly, the intracellular function of ANGPTL8 has been reported. It can enhance the cleavage of ANGPTL3, a molecule involved in the triglyceride metabolism ${ }^{20}$; and it is also involved in the lipolysis of adipocytes ${ }^{42}$.

ANGPTL8 has been reported to be induced by thyroid hormone, and modulates autophagy mainly via enhancing autolysosome maturation ${ }^{43}$. In this study, we found that ANGPTL8 specifically mediates the autophagic IKK $\gamma$ degradation, which can be promoted by overexpression of K63-linked ubiquitin, and chemical or genetic blockage of autophagy could inhibit this IKK $\gamma$ degradation (Figs. 5-7). Furthermore, p62, a classical autophagy receptor, works cooperatively with ANGPTL8 to mediate the proteolysis of IKK $\gamma$ (Figs. 7 and 8 and Supplementary Fig. 7). Although it has been reported that IKK $\gamma$ is mainly degraded by lysosomal pathway ${ }^{44}$, this is the first report that IKK $\gamma$ can be degraded by ANGPTL8/p62-mediated selective autophagy.

The specificity of selective autophagy for the degraded cargoes is mainly attributed to autophagy receptors. While there are numerous molecules or cell organelles need to be degraded, only a handful of known autophagy receptors are responsible for this process $^{15,31,45}$. There is a general model behind the selectivity: the substrate needs to be recognized by other proteins (e.g., molecular chaperones) before interacting with autophagy receptors, which can be termed as "co-receptor" 31 . In this study, several lines of evidence indicate ANGPTL8 as a co-receptor of p62 to mediate the IKK $\gamma$ selection. First, p62 and many other chaperones are stress-responsive, possibly to ensure different substrates are properly degraded under certain conditions ${ }^{46,47}$; similarly, ANGPTL8 is a stress-responsive molecule with enhanced expression under TNF $\alpha$, IL-1 $\beta$, LPS, or infection (Figs. 1 and 10 and Supplementary Fig. 1). Second, the interactions between p62 and ANGPTL8 are mutually required for IKK $\gamma$ degradation (Fig. 7). Third, TNF $\alpha$ treatment promotes the formation of the IKK $\gamma$-p62-ANGPTL8 complex, which is impaired in the ANGPTL8 knockdown or knockout cells (Fig. 8). Consistently, enhanced binding affinity between Ikk $\gamma$, Angptl8, and p62 in the liver of LPS-treated mice was also observed (Fig. 10d). Our results thus suggested a novel role of ANGPTL8 as a co-receptor of p62 in selective autophagy.

After cargo selection, an important role for autophagy receptors such as p62 is to sequester cargoes into larger aggregates 
a

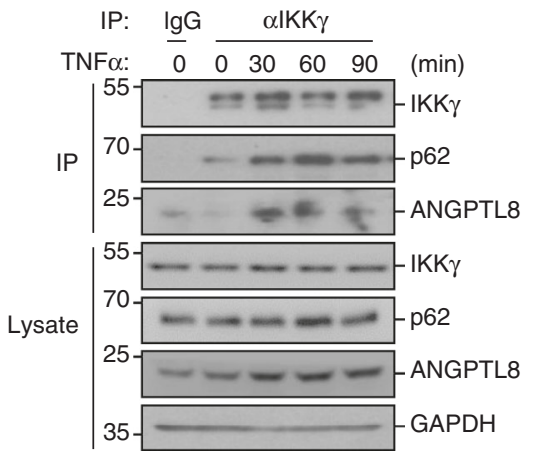

C

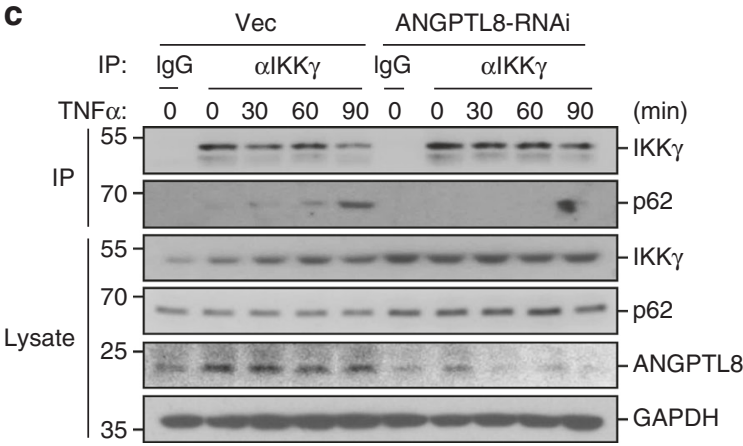

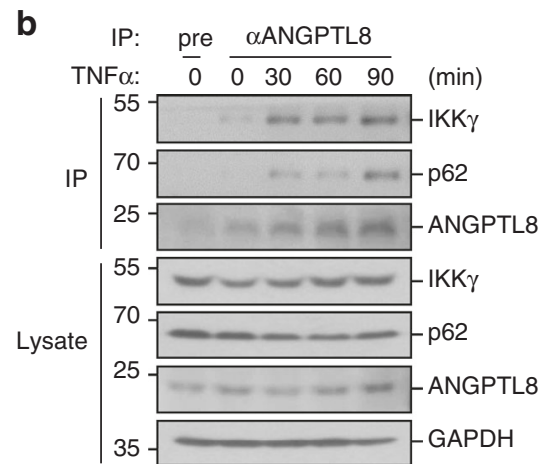

d

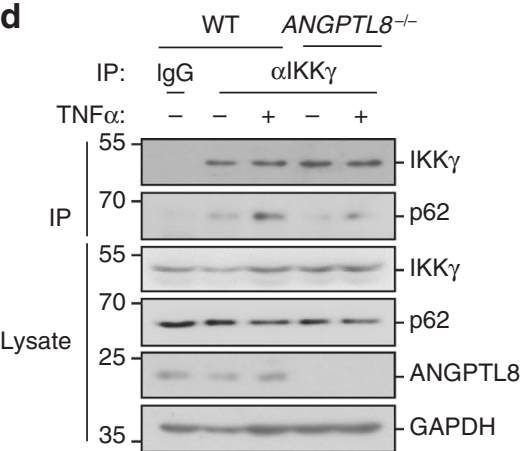

Fig. 8 ANGPTL8 is important for the IKK $\gamma$-p62 interaction. a, b ANGPTL8 forms a complex with IKK $\gamma$ and p62 upon TNF $\alpha$ stimulation. HepG2 cells were stimulated with TNF $\alpha$ or left unstimulated for indicated time $\left(2.5 \times 10^{7}\right.$ cells per group). And then cells were lysed and cell lysate was subjected to co-IP analysis by anti-IKK $\gamma$. (a) or anti-ANGPTL8 (b) followed immunoblots. $\mathbf{c}$ The effects of ANGPTL8-RNAi on the IKK $\gamma$-related protein complex formation. The experiment was performed as in a except the ANGTPL8-RNAi or control cells $\left(2.5 \times 10^{7}\right)$ were used. $\mathbf{d}$ The effects of ANGPTL8 deficiency on the IKK $\gamma$ related protein complex formation. ANGPTL8-deficient or control cells $\left(2.5 \times 10^{7}\right)$ were left untreated or treated with TNF $\alpha$ for $1 \mathrm{~h}$ before subjected to CoIP analysis and immunoblots. Data are representative of three independent experiments

before they being degraded ${ }^{31,48,49}$. During this process, p62 forms oligomers, and such suprastructure enables its interaction with LC3B and ubiquitinated cargoes ${ }^{49,50}$. It is an interesting and open question that whether other p62-related proteins work in similar ways $^{50}$. Here, we demonstrate that ANGPTL8 self-oligomerizes through its $\mathrm{N}$-terminal region and such oligomerization is essential for the interaction/degradation of IKK $\gamma$, thereby affecting the ANGPTL8-mediated inhibition of TNF $\alpha$-induced NF- $\mathrm{\kappa B}$ activation (Fig. 9), indicating the oligomerization is important to the interaction between the co-receptors and cargoes. However, ANGPTL8 seems to directly interact with p62, since ANGPTL8$\Delta 26-70$ showed stronger interaction with p62 compared with the full-length ANGPTL8 (Fig. 9).

As major protein degradation pathways, UPS and autophagy have been proven to play significant roles in inflammation ${ }^{17,18}$. While there have been dozens of regulators identified to influence NF- $\kappa B$ activation by UPS, reports on how autophagy degrades specific signaling molecules in inflammation are rare ${ }^{50-53}$, which may in part due to the concept that UPS is highly selective but autophagy is a bulk process. However, recent studies suggested both process can be specific, UPS is involved in the rapid degradation of single proteins, while autophagy can selectively remove protein aggregates and damaged/excess organelles that are too big in size for proteasomes ${ }^{14}$. Interestingly, signaling components, especially receptors and scaffold proteins, tend to form oligomers to mediate the signaling ${ }^{54}$, implicating that these aggregation-prone proteins may be the appropriate substrates for autophagy. IKK $\gamma$ is a well-known scaffold protein and TNF $\alpha$ stimulation can induce the IKK $\gamma$ translocation to supramolecular structures which is important to the NF- $\mathrm{KB}$ activation $4,5,27,55$. Here, we demonstrated that IKK $\gamma$ could undergo the ANGPTL8/ p62-facilitated selective autophagy, we also observed that the percentage of punctual IKK $\gamma$ can be enhanced by ANGPTL8, these findings suggested a possibility that aggregated IKK $\gamma$ is not only important for signaling transduction, but also a precondition for its degradation. Moreover, our study implicates that additional scaffold proteins in this or other pathways may also be degraded with similar mechanism.

Multiple studies have confirmed the induction of circulating ANGPTL8 in different metabolic syndromes ${ }^{36,37}$. Here we found that the level of circulating ANGPTL8 was dramatically increased in severe infection (Fig. 10e). To our knowledge, it is the first report on the relationship between ANGPTL8 and acute inflammation in clinical patients. We also observed a correlation of Angptl8 level with LPS-induced acute inflammatory response in different tissues of mouse (Fig. 10a and Supplementary Fig. 11). Future experiments using Angptl8 knockout or transgenic mice will be helpful to reveal the mechanisms underlying its physiological and pathological roles in inflammatory diseases.

Collectively, our results uncover an important fine-regulation mechanism for NF-KB activation. Notably, the observations that ANGPTL8 also can be induced by additional factors such as IL$1 \beta$, LPS, insulin resistance and feeding from this study and from literature ${ }^{56}$, implicate a more broaden role of ANGPTL8 in the autophagic degradation of other inflammation or metabolism associated proteins, a question awaits further exploration.

\section{Methods}

Reagents, antibodies and cell lines. Immunoblots with ANGPTL8/Angptl8 were done by mouse anti-ANGPTL8 monoclonal antibody (1:300), a kind gift from Dr. Y. Wang (Wuhan University) ${ }^{19,20}$. Immunofluorescence with ANGPTL8 was done by an anti-ANGPTL8 monoclonal antibody (Sigma, SAB3501080, 1:100). Co-IP 
a

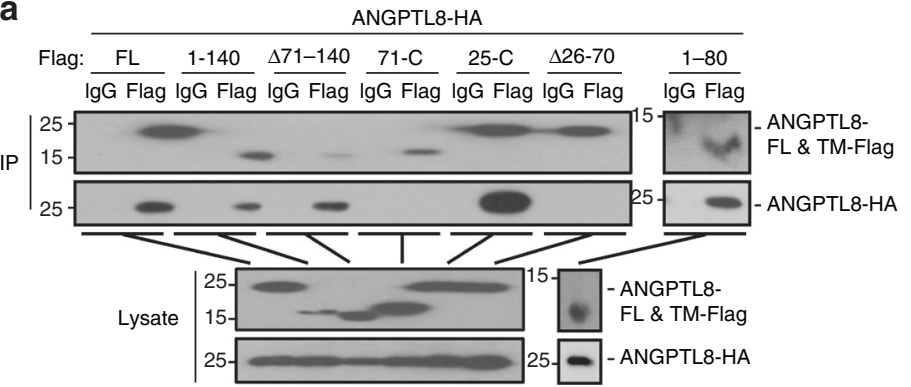

b

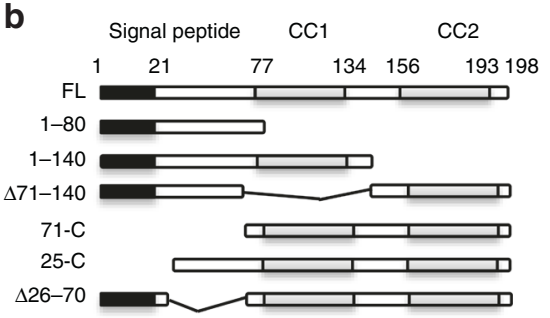

C

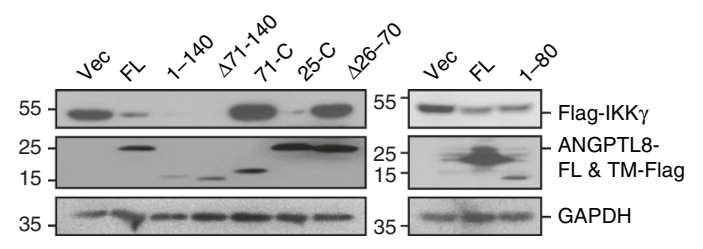

d

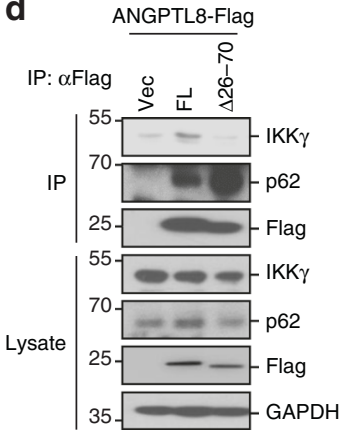

e

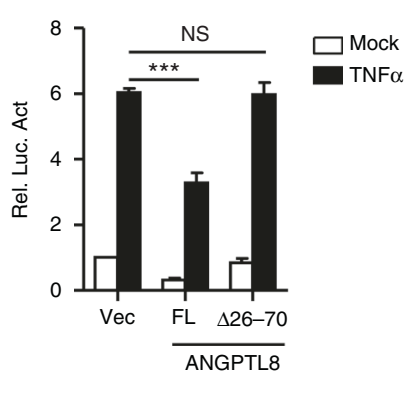

f

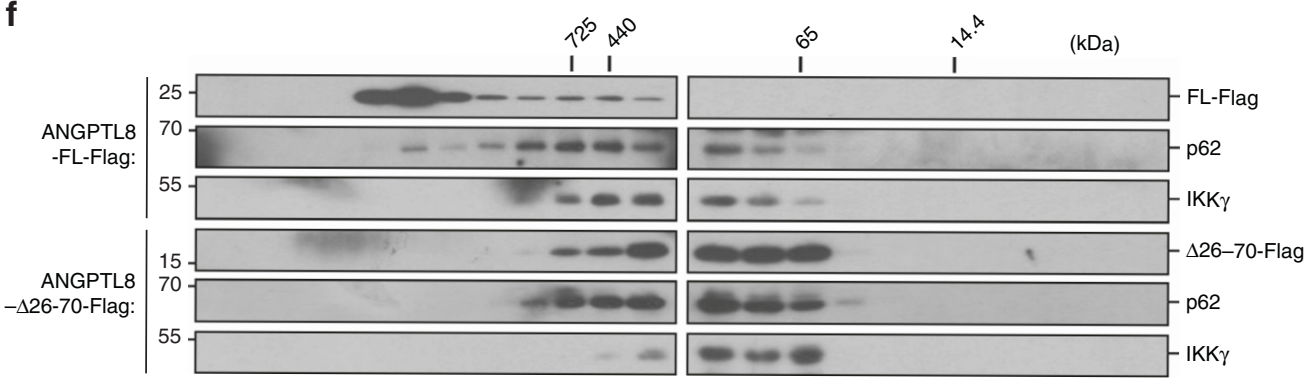

Fig. 9 ANGPTL8 self-aggregation is essential for the degradation of IKK $\gamma$. a Domain mapping of the ANGPTL8 self-aggregation in HEK293T cells. b A schematic presentation of human ANGPTL8 and its truncation mutants. $\mathbf{c}$ The effects of ANGPTL8 and its truncation mutants on the degradation of IKK $\gamma$ in HEK293T cells. d The ANGPTL8- $\Delta 26-70$ shows a diminished interaction with IKK $\gamma$. Three dishes of HEK293T cells $\left(2 \times 10^{6}\right.$ cells per dish $)$ were transfected with indicated plasmids, $24 \mathrm{~h}$ later, cells were lysed and subjected to co-IP and followed immunoblots by indicated antibodies. e The effects of the full-length or $-\Delta 26-70$ truncation mutant of ANGPTL8 on the TNF $\alpha$-induced NF-kB activation $(n=3)$. $\mathbf{f}$ Analysis of protein complex containing ANGPTL8, p62 and IKK $\gamma$ by size-exclusion chromatography. HEK293T cells $\left(2 \times 10^{6}\right)$ were transfected with indicated plasmids for $24 \mathrm{~h}$ later before cells were lysed and subjected to size-exclusion chromatography. Data are shown as the mean \pm SEM in e, unpaired two-tailed Student's test was used for statistics $(\mathbf{e}) .{ }^{* \star \star} p<0.0001, N S>0.05$. Data are representative of three independent experiments. FL full length, TM truncation mutants

experiments with ANGPTL8/Angptl8 were done by mouse anti-ANGPTL8 polyclonal antibody which was raised against recombinant human full-length ANGPTL8 with standard protocols. A list of commercial reagents, and other antibodies and dilutions used in the present study was provided in Supplementary Table 2. HepG2 (CL-0103000), HEK293T (CL-0005), and A549 (CL-0016) cells, which were analyzed with authenticated STR locus and tested for mycoplasma contamination, were purchased from Procell Biotech. (Wuhan, China).

Constructs. NF- $\mathrm{KB}$, IRF1 and TK luciferase reporter plasmids, mammalian expression plasmids for Flag-tagged RIP1, MyD88, TRAF6, IKK $\alpha$, IKK $\beta$, IKK $\gamma$ and p65; HA-tagged Ubi (WT, K63), prepared as previously described ${ }^{57,58}$, were kind gifts of Dr. H.-B. Shu (Wuhan University). EGFP tagged OPTN were purchased from Addgene (\#27052). CYLD-Flag is a kind gift of Dr. B. Zhong (Wuhan University). Lentiviral GFP-LC3 is a kind gift from Dr. Z.Y. Song (Wuhan University); Flag- or HA-tagged IKK $\gamma$; ANGPTL8 and their truncated mutants; HA-tagged p62, NDP52, and Tollip; Flag-tagged NBR1, p62, p62 LLIR and Cherry-tagged IKK $\gamma$ were constructed with standard procedures.

Transfection and luciferase reporter gene assays. Cells $\left(5 \times 10^{4}\right)$ were seeded on 48-well plates and transfected on the following day, empty control plasmid was added to ensure that each transfection receives the same amount of total DNA. To normalize transfection efficiency, $0.02 \mu \mathrm{g}$ of pRL-TK Renilla luciferase reporter plasmid was added to each transfection. Luciferase assays were performed using a dual-specific luciferase assay kit (Promega), the firefly luciferase activities (NF- $\mathrm{\kappa B}$ or IRF1 firefly luciferase reporter) were normalized based on Renilla luciferase activities.

RNAi experiments. Double-strand oligonucleotides corresponding to the target sequences were cloned into the pSuper plasmids (Oligoengine). The target sequences for human ANGPTL8, p62, IKK $\gamma$, ATG5, and ATG7 cDNA are listed in Supplementary Table 3.

Retrovirus-mediated stable RNAi cell lines. The packaging cell line HEK293T was transfected with retroviral vectors by calcium phosphate precipitation. Twelve hours later, cells were washed by PBS, and antibiotics-free medium was added for another $24 \mathrm{~h}$. The filtered supernatant was used to infect HepG2 or ANGPTL $8^{-1-}$ cells in the presence of $6 \mu \mathrm{g} / \mathrm{mL}$ polybrene. The infection was repeated twice to enhance transduction efficiency.

Quantitative real-time PCR. Total RNA was isolated from cells using RNAiso Plus reagent (Takara) and subjected to qPCR analysis. The mRNA levels of specific genes were normalized to GAPDH. The gene-specific primer sequences for ANGPTL8, IKKG, Angptl8, and Tnfa are listed in Supplementary Table 3. The 
a

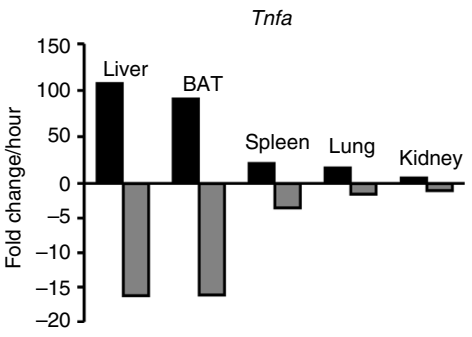

b

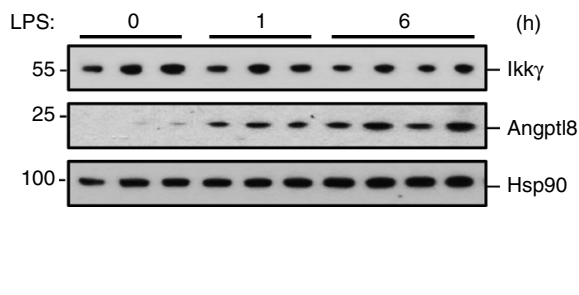

C

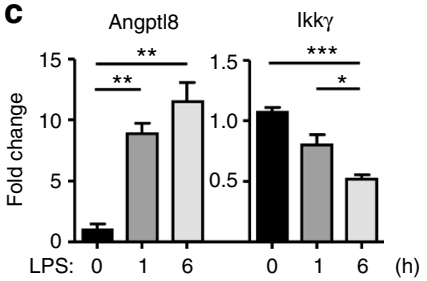

d

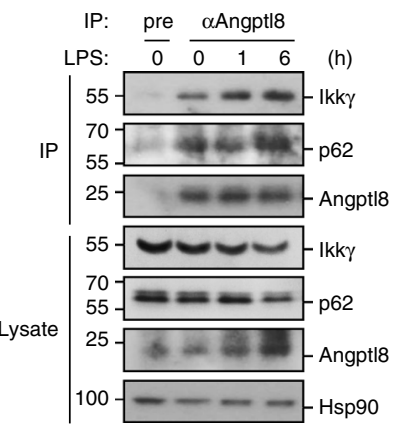

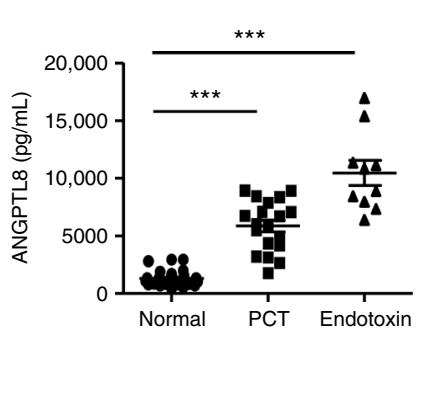

f

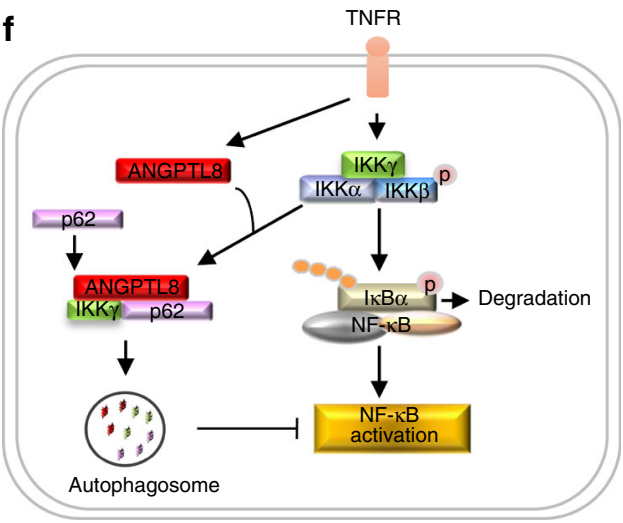

Fig. 10 Potential involvement of ANGPTL8 in the acute inflammation caused by infection. a The upregulation and downregulation rate of Tnfa transcription during the acute phase ( $0-1 \mathrm{~h})$ and the resolution phase (1-6h) upon LPS infection. The upregulation rate was determined by the fold change between 0-1 h, while the downregulation rate was determined by the fold change per hour between $1-6 \mathrm{~h}$ after the injection. ( $n=3$ for 0 and $1 \mathrm{~h}, n=4$ for $6 \mathrm{~h}$ ). b, c The immunoblots (b) and quantitation results (c) of the expression of Ikk $\gamma$ and Angptl8 in the liver upon LPS infection. Each lane stands for one mouse. d Angptl8 forms a complex with lkk $\gamma$ and p62 upon LPS infection. Equal amount of lysate obtained from each animal at the same time point were mixed, and subjected to Co-IP experiment ( $n=3$ for 0 and $1 \mathrm{~h}, n=4$ for $6 \mathrm{~h}$ ). e The circulating ANGPTL8 was elevated in patients with positive detection of PCT or endotoxin (healthy control, $n=30 ;$ PCT, $n=18$; endotoxin, $n=10$ ). f A working model of ANGTPL8 mediated regulation of TNF $\alpha$-induced signaling. TNF $\alpha$ upregulates the expression of ANGPTL8, and then aggregated ANGPTL8 facilitates the ANGPTL8-p62-IKK $\gamma$ complex formation, which promotes the autophagic IKK $\gamma$ degradation and inhibits TNF $\alpha$-induced NF-KB activation. Data are shown as the mean \pm SEM in $\mathbf{c}$, e, unpaired two-tailed Student's test was used for statistics. ${ }^{\star \star \star} p<0.0001,{ }^{\star \star} p<0.01,{ }^{\star} p<0.05$

primers for CXCL2, IL8, NFKBIA, TNFA, and GAPDH were as we previously described $^{58,59}$

CRISPR-Cas9-mediated knockout of ANGPTL8. The CRISPR-Cas9 based protocols for genome engineering were used as described ${ }^{60}$. PGL-U6-GRNA and PST1374-Cas9 plasmids were gifts of Dr. X. Zhang (Wuhan University). The ANGPTL8 gRNA target sequence and the identification primers for ANGPTL8 knockout are listed in Supplementary Table 3.

Confocal microscopy. The WT and ANGPTL $8^{-1-}$ stable cell lines expressing GFPLC3 were constructed by lentivirus mediated gene transfer. At $20 \mathrm{~h}$ after transfected with IKK $\gamma$-Cherry, cells were treated with or without TNF $\alpha$ for $2 \mathrm{~h}$. After fixing with $4 \%(\mathrm{~W} / \mathrm{V})$ formaldehyde, the nuclei were stained by DAPI $(1 \mu \mathrm{g} / \mathrm{mL})$ and the cells were imaged with a Zeiss LSM 880 confocal microscope. The plugin JACoP of Image J was used to calculate the colocalization rate for the red pixels and green dots (green dots represent LC3-II, a marker for autophagosome ${ }^{61}$ ) as described ${ }^{62}$. Colocalization of signals from IKK $\gamma$-Cherry and GFP-LC3 dots was evaluated using Manders' overlap coefficient and the Pearson's correlation coefficient.

Coimmunopreicipitation assays. Transfected HEK293T cells $\left(\sim 5 \times 10^{6}\right)$ or HepG2 cells $\left(\sim 2 \times 10^{7}\right)$ were lyzed in $1 \mathrm{~mL}$ pre-lysis buffer $(20 \mathrm{mM}$ Tris- $\mathrm{HCl}, \mathrm{pH}$ 7.4, $150 \mathrm{mM} \mathrm{NaCl}, 1 \mathrm{mM}$ EDTA, $1 \%$ Triton X-100, $10 \mu \mathrm{g} / \mathrm{mL}$ aprotinin, $10 \mu \mathrm{g} / \mathrm{mL}$ leupeptin, $0.5 \mathrm{mM} \beta$-glycerophosphate disodium salt hydrate and $1 \mathrm{mM}$ phenylmethylsulfonyl fluoride). For each immunoprecipitation, $0.8 \mathrm{~mL}$ of cell lysate was incubated with $0.5 \mu \mathrm{g}$ of the indicated antibody and $35 \mu \mathrm{L}$ of $50 \%$ slurry of GammaBind Plus-Sepharose (Amersham Biosciences) at $4{ }^{\circ} \mathrm{C}$ for $4 \mathrm{~h}$. The Sepharose beads were then washed three times with $1 \mathrm{~mL}$ of lysis buffer containing $500 \mathrm{mM} \mathrm{NaCl}$. The precipitates were resuspended by $60 \mu \mathrm{L}$ SDS loading buffer, and subsequent immunoblot analysis was performed with indicated antibodies.

Size-exclusion chromatography. Cells $\left(2 \times 10^{6}\right)$ were transfected with indicated plasmids for $24 \mathrm{~h}$ before being lysed in $500 \mu \mathrm{L}$ of pre-lysis buffer. The cell lysate was then incubated on ice for $30 \mathrm{~min}$ followed by sonication and was spun down at
$12000 \times g$ for $10 \mathrm{~min}$. The supernatant was filtered with a $0.45 \mu \mathrm{m}$ filter (Millipore) before being loaded onto a Superose 6 size-exclusion chromatography column (GE Healthcare, $1 \times 30 \mathrm{~cm}$ ), which was pre-equilibrated with Triton and EDTA free pre-lysis buffer. The samples were eluted at $4{ }^{\circ} \mathrm{C}$ by lysis buffer at a flow rate of 500 $\mu \mathrm{L} / \mathrm{min}$ and collected in fractions of $500 \mu \mathrm{L}$. The fractions were precipitated with $20 \%$ trichloroacetic acid and analyzed by immunoblots with antibodies against Flag, IKK $\gamma$, and p62.

Human studies. To compare circulating ANGPTL8 levels between patients with inflammation and healthy controls, patients with positive detection of procalcito$\operatorname{nin}(\mathrm{PCT}>0.5 \mu \mathrm{g} / \mathrm{L}, n=18$ ), positive detection of endotoxin (LPS $>0.1 \mathrm{EU} / \mathrm{mL}$, $n=10)$ and healthy controls $(n=30$, from physical examination center) were included in the study. The detail sample information was listed in Supplementary Table 1, Circulating levels of human ANGPTL8 were determined by enzyme immunoassay kit (EIAab Science, Wuhan, China). Informed consent was obtained from all subjects and the Ethical approval ((2017)09) was obtained by the Medical Ethics Committee of the Wuhan Hospital of Traditional and Western Medicine (Wuhan First Hospital).

Mice. Male C57BL/6 mice were obtained from the Center for Animal Experiment/ Animal Biosafety Level-III Laboratory of Wuhan University. Mice were housed in ventilated microisolator cages with free access to water and normal chow. Animals were handled according to the Guidelines of the China Animal Welfare Legislation, as approved by the Committee on Ethics in the Care and Use of Laboratory Animals of College of Life Sciences, Wuhan University. For LPS injection experiment, two-month-old mice were randomly divided into three groups and intraperitoneally (i.p.) injected with a single dose of LPS $(3 \mathrm{mg} / \mathrm{kg})$ for 1 or $6 \mathrm{~h}$, untreated age- and sex-matched littermate as controls $(n=3$ for 0 and $1 \mathrm{~h}, n=4$ for $6 \mathrm{~h}$ ). The mice were killed, liver, brown fat, spleen, lung, and kidney were collected.

Far-UV circular dichroism and structural modeling. A JASCO-810 circular dichroism spectropolarimeter (Tokyo, Japan) was used to monitor secondary structures. Incubated samples were diluted to a final concentration of $10 \mu \mathrm{M}$ and detected in a $1 \mathrm{~mm}$ path length at $25^{\circ} \mathrm{C}$. Circular dichroism (CD) spectra were 
obtained from 260 to $200 \mathrm{~nm}$ at a $200 \mathrm{~nm} /$ min scanning speed and a $2 \mathrm{~nm}$ bandwidth. All samples were measured in triplicates and the averages were taken. The data were converted to mean residue ellipticity and the secondary structural contents were further calculated with the software CDPro ${ }^{63}$. Structural modeling of ANGPTL8 was conducted using the online server I-TASSER.

Dynamic light scattering analysis. The sizes of ANGPTL8 aggregates were measured by dynamic light scattering in a zeta pals potential analyzer (Brookhaven Instruments, USA). Samples were vortexed and detected at room temperature, the scattering angle was set at $90^{\circ}$. Each measurement was repeated three times and the average mean particle size was recorded. The data was analyzed by the multimodal size distribution (MSD) software ${ }^{64}$.

Dot blot assays. Sample aliquotes $(2 \mu \mathrm{L})$ obtained at indicated time points were blotted onto a nitrocellulose membrane (Bio-Rad, USA). Dried membrane was blocked with $5 \%$ non-fat milk for $1 \mathrm{~h}$ at room temperature and then incubated with anti-oligomer antibody (A-11) or anti-fibril antibody (OC) at $4{ }^{\circ} \mathrm{C}$ overnight. The membrane was incubated with anti-rabbit IgG for $2 \mathrm{~h}$ at room temperature later. An ECL chemiluminescence kit (Advansta, USA) was used for the development.

Transmission electronic microscopy. Incubated solution was applied onto a 300mesh formvar-carbon coated copper grid and sit for $5 \mathrm{~min}$. Freshly prepared uranyl formate (1\%) was dropwise added for staining. Dried samples were observed under a transmission microscope (Hitachi, Japan) operating at an accelerating voltage of $200 \mathrm{kV}^{65}$.

Statistical analysis. Sample sizes, as described in figure legends, were selected based on effect size and availability as per usual standard. Randomization was done by selecting animals of similar age and weight. No blinding was involved in animal studies. Statistically significant differences between the mean values were determined by two-tailed Student's $t$-test $\left.{ }^{*} p<0.05,{ }^{* *} p<0.01,{ }^{* * *} p<0.001, \mathrm{NS}>0.05\right)$. Data are presented as the mean \pm SEM.

Data availability. The data that support the findings of this study is provided in the supplementary information (Supplementary Figs. 12-22) or available upon request.

Received: 17 January 2017 Accepted: 22 November 2017 Published online: 18 December 2017

\section{References}

1. Vallabhapurapu, S. \& Karin, M. Regulation and function of NF-kappaB transcription factors in the immune system. Annu. Rev. Immunol. 27, 693-733 (2009).

2. Scheidereit, C. IkappaB kinase complexes: gateways to NF-kappaB activation and transcription. Oncogene 25, 6685-6705 (2006).

3. Hacker, H. \& Karin, M. Regulation and function of IKK and IKK-related kinases. Sci. STKE 2006, re13 (2006).

4. Hayden, M. S. \& Ghosh, S. Shared principles in NF-kappaB signaling. Cell 132, 344-362 (2008).

5. Schrofelbauer, B., Polley, S., Behar, M., Ghosh, G. \& Hoffmann, A. NEMO ensures signaling specificity of the pleiotropic IKKbeta by directing its kinase activity toward IkappaBalpha. Mol. Cell 47, 111-121 (2012).

6. Yamamoto, Y. \& Gaynor, R. B. Role of the NF-kappaB pathway in the pathogenesis of human disease states. Curr. Mol. Med. 1, 287-296 (2001).

7. Tak, P. P. \& Firestein, G. S. NF-kappaB: a key role in inflammatory diseases. J. Clin. Invest. 107, 7-11 (2001).

8. Inoue, J., Gohda, J., Akiyama, T. \& Semba, K. NF-kappaB activation in development and progression of cancer. Cancer Sci. 98, 268-274 (2007).

9. Doffinger, R. et al. X-linked anhidrotic ectodermal dysplasia with immunodeficiency is caused by impaired NF-kappaB signaling. Nat. Genet. 27, 277-285 (2001)

10. Courtois, G., Smahi, A. \& Israel, A. NEMO/IKK gamma: linking NF-kappa B to human disease. Trends Mol. Med. 7, 427-430 (2001).

11. Malynn, B. A. \& Ma, A. A20 takes on tumors: tumor suppression by an ubiquitin-editing enzyme. J. Exp. Med. 206, 977-980 (2009).

12. Sun, S. C. CYLD: a tumor suppressor deubiquitinase regulating NF-kappaB activation and diverse biological processes. Cell Death Differ. 17, 25-34 (2010).

13. Zilberman-Rudenko, J. et al. Recruitment of A20 by the C-terminal domain of NEMO suppresses NF-kappaB activation and autoinflammatory disease. Proc. Natl Acad. Sci. USA 113, 1612-1617 (2016).

14. Kraft, C., Peter, M. \& Hofmann, K. Selective autophagy: ubiquitin-mediated recognition and beyond. Nat. Cell Biol. 12, 836-841 (2010).
15. Stolz, A., Ernst, A. \& Dikic, I. Cargo recognition and trafficking in selective autophagy. Nat. Cell Biol. 16, 495-501 (2014).

16. Mizushima, N., Yoshimori, T. \& Ohsumi, Y. The role of Atg proteins in autophagosome formation. Annu. Rev. Cell Dev. Biol. 27, 107-132 (2011).

17. Ma, Y., Galluzzi, L., Zitvogel, L. \& Kroemer, G. Autophagy and cellular immune responses. Immunity 39, 211-227 (2013).

18. Zhong, Z., Sanchez-Lopez, E. \& Karin, M. Autophagy, inflammation, and immunity: a troika governing cancer and its treatment. Cell 166, 288-298 (2016).

19. Wang, Y. et al. Mice lacking ANGPTL8 (Betatrophin) manifest disrupted triglyceride metabolism without impaired glucose homeostasis. Proc. Natl Acad. Sci. USA 110, 16109-16114 (2013).

20. Quagliarini, F. et al. Atypical angiopoietin-like protein that regulates ANGPTL3. Proc. Natl Acad. Sci. USA 109, 19751-19756 (2012).

21. $\mathrm{Fu}, \mathrm{Z}$. et al. Elevated circulating lipasin/betatrophin in human type 2 diabetes and obesity. Sci. Rep. 4, 5013 (2014)

22. Li, S. et al. Circulating Betatrophin in Patients with Type 2 Diabetes: A MetaAnalysis. J. Diabetes Res. 2016, 6194750 (2016).

23. Chi X. et. al. ANGPTL8 promotes the ability of ANGPTL3 to bind and inhibit lipoprotein lipase. Mol. Metab. 6, 1137-1149 (2017).

24. Kuma, A. et al. The role of autophagy during the early neonatal starvation period. Nature 432, 1032-1036 (2004).

25. Komatsu, M. et al. Impairment of starvation-induced and constitutive autophagy in Atg7-deficient mice. J. Cell Biol. 169, 425-434 (2005).

26. Tarantino N. et al. TNF and IL-1 exhibit distinct ubiquitin requirements for inducing NEMO-IKK supramolecular structures. J. Cell Biol. 204, 231-245 (2014).

27. Wacker, C., Prkno, A., Brunkhorst, F. M. \& Schlattmann, P. Procalcitonin as a diagnostic marker for sepsis: a systematic review and meta-analysis. Lancet Infect. Dis. 13, 426-435 (2013).

28. Kovalenko, A. et al. The tumour suppressor CYLD negatively regulates NFkappaB signalling by deubiquitination. Nature 424, 801-805 (2003).

29. Wang, D. W., Peng, Z. J., Ren, G. F. \& Wang, G. X. The different roles of selective autophagic protein degradation in mammalian cells. Oncotarget 6 37098-37116 (2015).

30. Pankiv, S. et al. p62/SQSTM1 binds directly to Atg8/LC3 to facilitate degradation of ubiquitinated protein aggregates by autophagy. J. Biol. Chem. 282, 24131-24145 (2007).

31. Johansen, T. \& Lamark, T. Selective autophagy mediated by autophagic adapter proteins. Autophagy 7, 279-296 (2011).

32. Siddiqa, A. et al. Structural characterization of ANGPTL8 (betatrophin) with its interacting partner lipoprotein lipase. Comput. Biol. Chem. 61, 210-220 (2016)

33. Santulli, G. Angiopoietin-like proteins: a comprehensive look. Front. Endocrinol. 5, 4 (2014)

34. Hurley, J. C., Guidet, B., Offenstadt, G. \& Maury, E. Endotoxemia and mortality prediction in ICU and other settings: underlying risk and co-detection of gram negative bacteremia are confounders. Crit. Care 16, R148 (2012).

35. Fu, Z., Abou-Samra, A. B. \& Zhang, R. A lipasin/Angptl8 monoclonal antibody lowers mouse serum triglycerides involving increased postprandial activity of the cardiac lipoprotein lipase. Sci. Rep. 5, 18502 (2015).

36. Abu-Farha, M. et al. Higher plasma betatrophin/ANGPTL8 level in Type 2 Diabetes subjects does not correlate with blood glucose or insulin resistance. Sci. Rep. 5, 10949 (2015)

37. Lee, Y. H. et al. Association between betatrophin/ANGPTL8 and non-alcoholic fatty liver disease: animal and human studies. Sci. Rep. 6, 24013 (2016)

38. Bogunovic, D. et al. Mycobacterial disease and impaired IFN-gamma immunity in humans with inherited ISG15 deficiency. Science 337, 1684-1688 (2012).

39. Zhang, X. et al. Human intracellular ISG15 prevents interferon-alpha/beta over-amplification and auto-inflammation. Nature 517, 89-93 (2015).

40. Lagace, T. A. et al. Secreted PCSK9 decreases the number of LDL receptors in hepatocytes and in livers of parabiotic mice. J. Clin. Invest. 116, 2995-3005 (2006).

41. Poirier, S. et al. Dissection of the endogenous cellular pathways of PCSK9induced low density lipoprotein receptor degradation: evidence for an intracellular route. J. Biol. Chem. 284, 28856-28864 (2009).

42. Mysore R., Liebisch G., Zhou Y., Olkkonen V. M., Nidhina Haridas P. A. Angiopoietin-like 8 (Angptl8) controls adipocyte lipolysis and phospholipid composition. Chem. Phys. Lipids 207, 246-252 (2017).

43. Tseng, Y. H. et al. Chromosome 19 open reading frame 80 is upregulated by thyroid hormone and modulates autophagy and lipid metabolism. Autophagy 10, 20-31 (2014)

44. Shibata, Y. et al. p47 negatively regulates IKK activation by inducing the lysosomal degradation of polyubiquitinated NEMO. Nat. Commun. 3, 1061 (2012).

45. Kirkin, V., McEwan, D. G., Novak, I. \& Dikic, I. A role for ubiquitin in selective autophagy. Mol. Cell 34, 259-269 (2009).

46. Szeto, J. et al. ALIS are stress-induced protein storage compartments for substrates of the proteasome and autophagy. Autophagy 2, 189-199 (2006) 
47. Gamerdinger, M. et al. Protein quality control during aging involves recruitment of the macroautophagy pathway by BAG3. EMBO J. 28, 889-901 (2009).

48. Lin, X. et al. Interaction domains of p62: a bridge between p62 and selective autophagy. DNA Cell Biol. 32, 220-227 (2013).

49. Ciuffa, R. et al. The selective autophagy receptor p62 forms a flexible filamentous helical scaffold. Cell Rep. 11, 748-758 (2015).

50. Wurzer, B. et al. Oligomerization of p62 allows for selection of ubiquitinated cargo and isolation membrane during selective autophagy. eLife 4, e08941 (2015).

51. Paul, S., Kashyap, A. K., Jia, W., He, Y. W. \& Schaefer, B. C. Selective autophagy of the adaptor protein Bcl10 modulates $\mathrm{T}$ cell receptor activation of NFkappaB. Immunity 36, 947-958 (2012).

52. Chang, C. P., Su, Y. C., Hu, C. W. \& Lei, H. Y. TLR2-dependent selective autophagy regulates NF-kappaB lysosomal degradation in hepatoma-derived M2 macrophage differentiation. Cell Death Differ. 20, 515-523 (2013).

53. Wertz, I. E. \& Dixit, V. M. Signaling to NF-kappaB: regulation by ubiquitination. Cold Spring Harb. Perspect. Biol. 2, a003350 (2010).

54. Napetschnig, J. \& Wu, H. Molecular Basis of NF-кB Signaling. Annu. Rev. Biophys. 42, 443-468 (2013).

55 . Weil, R. et al. Induction of the NF-kappaB cascade by recruitment of the scaffold molecule NEMO to the T cell receptor. Immunity 18, 13-26 (2003).

56. Zhang, R. The ANGPTL3-4-8 model, a molecular mechanism for triglyceride trafficking. Open Biol. 6, 150272 (2016).

57. Lei, C. Q. et al. Glycogen synthase kinase 3 beta regulates IRF3 transcription factor-mediated antiviral response via activation of the kinase TBK1. Immunity 33, 878-889 (2010).

58. Zhang, Y. et al. Kruppel-like factor 6 is a co-activator of NF-kappaB that mediates p65-dependent transcription of selected downstream genes. J. Biol. Chem. 289, 12876-12885 (2014).

59. $\mathrm{Hu}, \mathrm{M}$. M. et al. TRIM38 inhibits TNFalpha- and IL-1beta-triggered NFkappaB activation by mediating lysosome-dependent degradation of TAB2/3. Proc. Natl Acad. Sci. USA 111, 1509-1514 (2014).

60. Ran, F. A. et al. Genome engineering using the CRISPR-Cas9 system. Nat. Protoc. 8, 2281-2308 (2013)

61. Kabeya Y. et al. LC3, a mammalian homologue of yeast Apg8p, is localized in autophagosome membranes after processing. EMBO J. 19, 5720-5728 (2000).

62. Bolte, S. \& Cordelieres, F. P. A guided tour into subcellular colocalization analysis in light microscopy. J. Microsc. 224, 213-232 (2006).

63. Huang, K., Maiti, N. C., Phillips, N. B., Carey, P. R. \& Weiss, M. A. Structurespecific effects of protein topology on cross-beta assembly: studies of insulin fibrillation. Biochemistry 45, 10278-10293 (2006).

64. Gong, H. et al. Bisphenol A accelerates toxic amyloid formation of human islet amyloid polypeptide: a possible link between bisphenol A exposure and type 2 diabetes. PLOS ONE 8, e54198 (2013).
65. Cheng, B. et al. Silibinin inhibits the toxic aggregation of human islet amyloid polypeptide. Biochem. Biophys. Res. Commun. 419, 495-499 (2012).

\section{Acknowledgements}

We thank Drs. H.-B. Shu, B. Zhong, C.-Q. Lei, M.-M. Hu, H. Chen, and W.-J. Wang for technical help and stimulating discussions. This study was supported by the Natural Science Foundation of China (31500706, 31471208, and 31671195), Natural Science Foundation of Hubei Province (2015CFB245), the Fundamental Research Fund for the Central Universities (2016YXMS143), the Integrated Innovative Team for Major Human Diseases Program of Tongji Medical College, and the Front Youth Program of HUST.

\section{Author contributions}

Y.Z. and K.H. designed research; Y.Z., X.G., W.-Y.Y., Y.C., M.-X.K., C.C., X.-Q.Z., W.-L. X., Q.-Q. Z., L.M., and L.-Q.H. performed research; B.W., S.W., X.-R.L., and L.Z. contributed new reagents/analytic tools; Y.Z., X.G., W.-Y.Y., Y.C., C.C., L.Z., and K.H. analyzed data; and Y.Z., L.Z., and K.H. wrote the paper.

\section{Additional information}

Supplementary Information accompanies this paper at https://doi.org/10.1038/s41467 017-02355-w.

Competing interests: The authors declare no competing financial interests.

Reprints and permission information is available online at http://npg.nature.com/ reprintsandpermissions/

Publisher's note: Springer Nature remains neutral with regard to jurisdictional claims in published maps and institutional affiliations.

Open Access This article is licensed under a Creative Commons Attribution 4.0 International License, which permits use, sharing, adaptation, distribution and reproduction in any medium or format, as long as you give appropriate credit to the original author(s) and the source, provide a link to the Creative Commons license, and indicate if changes were made. The images or other third party material in this article are included in the article's Creative Commons license, unless indicated otherwise in a credit line to the material. If material is not included in the article's Creative Commons license and your intended use is not permitted by statutory regulation or exceeds the permitted use, you will need to obtain permission directly from the copyright holder. To view a copy of this license, visit http://creativecommons.org/ licenses/by/4.0/

(c) The Author(s) 2017 